MATHEMATICS OF COMPUTATION

Volume 75, Number 254, Pages 565-594

S $0025-5718(06) 01820-5$

Article electronically published on February 2, 2006

\title{
A NONLINEAR APPROACH TO ABSORBING BOUNDARY CONDITIONS FOR THE SEMILINEAR WAVE EQUATION
}

\author{
JÉRÉMIE SZEFTEL
}

\begin{abstract}
We construct a family of absorbing boundary conditions for the semilinear wave equation. Our principal tool is the paradifferential calculus which enables us to deal with nonlinear terms. We show that the corresponding initial boundary value problems are well posed. We finally present numerical experiments illustrating the efficiency of the method.
\end{abstract}

\section{INTRODUCTION}

The semilinear wave equation in $\mathbb{R}$ models various phenomena as the dislocation in crystals, laser pulses in two state media, etc. (see for instance 22]). Numerical computations of this equation are therefore often necessary. Though the problem is defined in the whole space, it is often sufficient to know the solution only on a bounded domain: the domain of interest. An artificial domain which includes this region of interest is then defined. Inside the domain the equations are discretized in the usual way, but there remains the question of the choice of reliable boundary conditions on the artificial boundary. Thus the boundary conditions have to be well posed and accurate in order to be able to approximate the restriction of the solution to the domain of interest.

In the case of linear constant coefficients equations, the transparent boundary condition (the boundary condition satisfied by the exact solution) can be explicitly computed when choosing special geometries for the computational domain (halfspaces, spheres or cylinders). This boundary condition is a nonlocal operator and can be approximated by local boundary conditions [13, 17, 21] or fast evaluated [8, 2], 9]. One can also replace the boundary condition by a reflectionless sponge layer damping propagating waves 3 .

In the case of linear variable coefficients equations, the previous methods fail since the transparent boundary condition cannot be explicitly computed. A strategy has been developed to design boundary conditions which minimize the reflection of the solution at the artificial boundary. These absorbing boundary conditions have been constructed for hyperbolic problems [7] and parabolic problems [10, with success, using pseudodifferential calculus. In the case of the wave equation, the method relies on the factorization of the operator in a product of two first-order

Received by the editor September 19, 2003 and, in revised form, April 27, 2005.

2000 Mathematics Subject Classification. Primary 35L70, 35S50, 35A21, 35A07, 65M99.

Key words and phrases. Semilinear wave equation, absorbing boundary conditions, paradifferential calculus. 
operators. One operator corresponds to the incident wave and the other to the reflected wave with respect to the boundary of the computational domain. As the boundary is artificial, the transparent boundary condition consists in annihilating the operator corresponding to the reflected wave. Finally, the transparent condition is not very manageable for numerical simulation and is approximated with absorbing boundary conditions, which are easy to implement.

For nonlinear problems, very little is known. To extend the strategy designed for linear variable coefficient equations to nonlinear equations, one of the first ideas is to use the absorbing boundary conditions of the linearized problem. We obtained good results in the case of reaction-diffusion equations [19] using this strategy. In the case of nonlinear Schrödinger equations, we showed in 20] that the solution computed with this method is very different from the solution of the nonlinear problem set on $\mathbb{R}$.

This motivates the introduction of a new method. It consists in following the same steps as in the linear case, replacing the pseudodifferential calculus with the paradifferential calculus. This enables us to deal with the nonlinear terms. E. Dubach [6] used this method in a formal way for nonlinear parabolic equations. Here, we develop this strategy in a rigorous way for the semilinear wave equation. The present work consists of six parts:

- First, we state the main results which will be established in the next sections.

- In section 3, we recall how to obtain the absorbing boundary conditions for the wave equation with variable coefficients following B. Engquist and A. Majda [7. We deduce a first way to construct absorbing boundary conditions for the nonlinear problem.

- In section 4, we recall the properties of the paradifferential calculus that we use in the sequel to find absorbing boundary conditions. We give various properties of the paradifferential calculus of J. M. Bony [4], then we recall various properties of the tangential paradifferential calculus of M. SabléTougeron [17].

- In section 5, we show that the solution of the semilinear wave equation also satisfies a paradifferential equation modulo a regular remainder. We factorize this paradifferential operator in a product of two first-order operators as in the linear case [7. Relying on a result of reflection of singularities, we show that the solution of the semilinear wave equation annihilates the first-order operator corresponding to the reflected wave modulo a regular remainder. This operator defines the transparent boundary condition. However, this boundary condition contains paradifferential operators which are nonlocal in time. Thus, giving local approximations of those operators, we find absorbing boundary conditions.

- In section 6 , we prove the well-posedness (local existence and uniqueness) for the semilinear wave equation with our absorbing boundary conditions. We study a linear nonhomogeneous problem which allows us to define an iterative scheme and show its convergence.

- In section 7, we numerically compute the solution of the semilinear wave equation with our absorbing boundary conditions. The schemes that we use are easy to implement. We show the qualities of the method and compare it with the one using pseudodifferential calculus. 
Remark. We study the one-dimensional case in order to emphasize the efficiency of our method in a simple case. The multidimensional study contains additional difficulties due to the geometry (see [10] in the case of linear parabolic equations on curved boundaries) and should be the heart of a forthcoming paper.

Remark. The reader more focused on applications may skip the rather technical sections 4 to 6 .

\section{Statement of the main Results}

Let $s$ be an integer such that $s>2$ and let $f$ in $C^{\infty}\left(\mathbb{R}^{3}, \mathbb{R}\right)$ be such that $f(0,0,0)=0$ and $f$ and all its derivatives are bounded. For all $u_{0}$ in $H^{s}(\mathbb{R})$ and for all $u_{1}$ in $H^{s-1}(\mathbb{R})$, there exists a time $T>0$ and a unique solution $u_{e x}$ in $L^{\infty}(] 0, T\left[, H^{s}(\mathbb{R})\right) \cap W^{1,+\infty}(] 0, T\left[, H^{s-1}(\mathbb{R})\right)$ of

$$
\left\{\begin{array}{l}
\left.\left(\partial_{t}^{2}-\partial_{x}^{2}\right) u_{e x}=f\left(u_{e x}, \partial_{t} u_{e x}, \partial_{x} u_{e x}\right) \text { in }\right] 0, T[\times \mathbb{R}, \\
u_{e x}=u_{0}, \partial_{t} u_{e x}=u_{1} \text { at } t=0 .
\end{array}\right.
$$

Moreover, $\partial^{\alpha} u_{e x}$ belongs to $L^{\infty}(] 0, T\left[, L^{2}(\mathbb{R})\right)$ for $|\alpha| \leq s$. Therefore, $u_{e x}$ is in $H^{s}(] 0, T[\times \mathbb{R})$. This result is proved for instance in [12].

We want to approximate the solution $u_{e x}$ of (2.1) restricted to $\mathbb{R}^{-}$. Suppose that $u_{0}$ and $u_{1}$ have support in $\mathbb{R}^{-}$. We look for a condition satisfied by $u_{e x}$ at $x=0$ of the form $\left.\left(\partial_{x}-B\right) u_{e x}\right|_{x=0}=0$, where $B\left(u_{e x}, t, D_{t}\right)$ is an operator. Then, $u_{e x}$ is the solution of the problem

$$
\left\{\begin{array}{l}
\left.\left(\partial_{t}^{2}-\partial_{x}^{2}\right) u_{e x}=f\left(u_{e x}, \partial_{t} u_{e x}, \partial_{x} u_{e x}\right) \text { in }\right] 0, T\left[\times \mathbb{R}^{-},\right. \\
\partial_{x} u_{e x}=B\left(u_{e x}, t, D_{t}\right) u_{e x} \text { at } x=0, \\
u_{e x}=u_{0}, \partial_{t} u_{e x}=u_{1} \text { at } t=0 .
\end{array}\right.
$$

Remark. In the case of linear equations, the operator $B$ does not depend of $u_{e x}$ (see for instance [7] and [10]).

We use two methods relying respectively on the pseudodifferential calculus and on the paradifferential calculus. These methods yield the absorbing boundary condition (abs) of order 0:

$$
\partial_{x} u+\partial_{t} u=0 \text { at } x=0 .
$$

We sum up the first-order and second-order absorbing boundary conditions given by the pseudodifferential strategy and the paradifferential strategy for functions $f$ of the type $f\left(u, u_{t}, u_{x}\right)=f_{1}(u)+f_{2}(u) u_{t}+f_{3}(u) u_{x}$, where $f_{j}$ is in $C^{\infty}(\mathbb{R}), 1 \leq j \leq 3$, and $f_{1}(0)=0$.

Pseudodifferential strategy. The first-order absorbing boundary condition is

$$
\left.\left(\partial_{x} u+\partial_{t} u-\left(f_{2}(u)-f_{3}(u)\right) u / 2\right)\right|_{x=0}=0,
$$

and the second-order absorbing boundary condition is

$$
\begin{aligned}
& \left(\partial_{t} \partial_{x} u+\partial_{t}^{2} u-1 / 2 f_{1}(u)\right. \\
& \quad+\left(f_{2}(u)^{2}-f_{3}(u)^{2}\right) u / 8+1 / 2\left(f_{3}(u)-3 f_{2}(u)\right) \partial_{t} u \\
& \left.\quad+\left(f_{3}^{\prime}(u)-f_{2}^{\prime}(u)\right)\left(\partial_{t} u+\partial_{x} u\right) u / 4\right)\left.\right|_{x=0}=0 .
\end{aligned}
$$


TABLE 1. First-order and second-order absorbing boundary conditions for $\left(\partial_{t}^{2}-\partial_{x}^{2}\right) u=f(u)$ at $x=0$.

\begin{tabular}{|c|c|c|}
\hline & pseudodifferential strategy & paradifferential strategy \\
\hline first-order abc & $\partial_{x} u+\partial_{t} u=0$ & $\partial_{x} u+\partial_{t} u=0$ \\
\hline second-order abc & $\partial_{t} \partial_{x} u+\partial_{t}^{2} u-f(u) / 2=0$ & $\partial_{t} \partial_{x} u+\partial_{t}^{2} u-f(u) / 2=0$ \\
\hline
\end{tabular}

TABLE 2. First-order and second-order absorbing boundary conditions for $\left(\partial_{t}^{2}-\partial_{x}^{2}\right) u=-u^{2} \partial_{t} u$ at $x=0$.

\begin{tabular}{|c|c|c|}
\hline & pseudodifferential strategy & paradifferential strategy \\
\hline first-order abc & $\partial_{x} u+\partial_{t} u+u^{3} / 2=0$ & $\partial_{x} u+\partial_{t} u+u^{3} / 6=0$ \\
\hline second-order abc & $\begin{array}{c}\partial_{t} \partial_{x} u+\partial_{t}^{2} u+\partial_{x} u u^{2} / 2 \\
+\partial_{t} u u^{2}-u^{5} / 8=0\end{array}$ & $\begin{array}{c}\partial_{t} \partial_{x} u+\partial_{t}^{2} u+3 \partial_{t} u u^{2} / 4 \\
+\partial_{x} u u^{2} / 4=0\end{array}$ \\
\hline
\end{tabular}

TABLE 3. First-order and second-order absorbing boundary conditions for $\left(\partial_{t}^{2}-\partial_{x}^{2}\right) u=u^{2} \partial_{x} u$ at $x=0$.

\begin{tabular}{|c|c|c|}
\hline & pseudodifferential strategy & paradifferential strategy \\
\hline first-order abc & $\partial_{x} u+\partial_{t} u+u^{3} / 2=0$ & $\partial_{x} u+\partial_{t} u+u^{3} / 6=0$ \\
\hline second-order abc & $\partial_{t} \partial_{x} u+\partial_{t}^{2} u+\partial_{x} u u^{2} / 2$ & $\partial_{t} \partial_{x} u+\partial_{t}^{2} u+\partial_{t} u u^{2} / 4$ \\
& $+\partial_{t} u u^{2}+u^{5} / 8=0$ & $-\partial_{x} u u^{2} / 4=0$ \\
\hline
\end{tabular}

Paradifferential strategy. The first-order absorbing boundary condition is

$$
\left.\left(\partial_{x} u+\partial_{t} u-F(u) / 2\right)\right|_{x=0}=0
$$

where $F$ is the primitive of $f_{2}-f_{3}$ vanishing at 0 . The second-order absorbing boundary condition is

$\left.\left(\partial_{t} \partial_{x} u+\partial_{t}^{2} u-1 / 2 f_{1}(u)+1 / 4\left(f_{3}(u)-3 f_{2}(u)\right) \partial_{t} u-1 / 4\left(f_{2}(u)+f_{3}(u)\right) \partial_{x} u\right)\right|_{x=0}=0$.

Using (2.4), (2.5), (2.6) and (2.7), we give the first-order and second-order absorbing boundary conditions obtained with the pseudodifferential and the paradifferential strategy for three examples of function $f$ studied in section 7 $f\left(u, \partial_{t} u, \partial_{x} u\right)=f(u), f\left(u, \partial_{t} u, \partial_{x} u\right)=-u^{2} \partial_{t} u$ and $f\left(u, \partial_{t} u, \partial_{x} u\right)=u^{2} \partial_{x} u$.

We obtain the same absorbing boundary conditions for the pseudodifferential and the paradifferential strategy, which is a coincidence, as we will see in the other examples.

Considering (2.2), we approximate $u_{e x}$ by the solution $u$ of

$$
\left\{\begin{array}{l}
\left.\left(\partial_{t}^{2}-\partial_{x}^{2}\right) u=f\left(u, \partial_{t} u, \partial_{x} u\right) \text { in }\right] 0, T\left[\times \mathbb{R}^{-}\right. \\
\partial_{x} u+\partial_{t} u+\partial_{t}^{-1}\left(f_{1}\left(u, \partial_{t} u, \partial_{x} u\right)\right)=0, \text { at } x=0 \\
u=u_{0}, \partial_{t} u=u_{1} \text { at } t=0
\end{array}\right.
$$

where $\partial_{t}^{-1} v=\int_{0}^{t} v(s) d s, f_{1}$ is a $C^{\infty}$ function in $\mathbb{R}^{3}$ such that $f_{1}(0,0,0)=0$, and $f_{1}$ corresponds to one of the boundary conditions (2.4), (2.5), (2.6) and (2.7). 
The following theorem shows that (2.8) is well posed.

Theorem 1. For all $u_{0}$ in $H^{2}\left(\mathbb{R}^{-}\right)$and $u_{1}$ in $H^{1}\left(\mathbb{R}^{-}\right)$with support in $\mathbb{R}^{-}$, there is a time $T>0$ such that problem (2.8) has a unique solution $u$ with $\partial^{\alpha} u$ in $L^{\infty}(] 0, T\left[, L^{2}\left(\mathbb{R}^{-}\right)\right)$for all $|\alpha| \leq 2$.

\section{ABSORBING BOUNDARY CONDITIONS USING PSEUDODIFFERENTIAL CALCULUS}

We first recall the strategy of B. Engquist and A. Majda [7] used to design absorbing boundary conditions for the wave equation with variable coefficients. Let $u_{0}$ and $u_{1}$ have compact support in $\mathbb{R}^{-}$, and let $u_{e x}$ be the solution of

$$
\left\{\begin{array}{l}
\left(\partial_{t}^{2}-\partial_{x}^{2}\right) u_{e x}+\alpha(t, x) u_{e x}+\beta(t, x) \partial_{t} u_{e x}+\gamma(t, x) \partial_{x} u_{e x}=0 \text { in } \mathbb{R}^{+} \times \mathbb{R} \\
u_{e x}=u_{0}, \partial_{t} u_{e x}=u_{1} \text { at } t=0
\end{array}\right.
$$

where $\alpha, \beta$ and $\gamma$ are $C^{\infty}$ functions in time and space. We want to approximate the restriction of $u_{e x}$ to $\mathbb{R}^{-}$. Therefore, we look for a condition satisfied by $u_{e x}$ at $x=0$ of the form $\left.\left(\partial_{x}-B\right) u_{e x}\right|_{x=0}=0$, where $B$ is an operator. We factorize the operator in (3.1) using L. Nirenberg's procedure [16]:

$$
\begin{aligned}
& \partial_{t}^{2}-\partial_{x}^{2}+\alpha(t, x)+\beta(t, x) \partial_{t}+\gamma(t, x) \partial_{x} \\
& =-\left(\partial_{x}-a\left(x, t, D_{t}\right)\right)\left(\partial_{x}-b\left(x, t, D_{t}\right)\right)+r\left(x, t, D_{t}\right),
\end{aligned}
$$

where $D_{t}=-i \partial_{t}, r$ is $C^{\infty}$ in $x$ with values in $S^{-\infty}\left(\mathbb{R}^{2}\right)$ defined by

$$
S^{-\infty}=\left\{q \in C^{\infty}\left(\mathbb{R}^{2}\right) /\left|\partial_{t}^{k} \partial_{\tau}^{l} q(t, \tau)\right| \leq C_{k l N}\left(1+\tau^{2}\right)^{-N}, \forall(k, l, N) \in \mathbb{N}^{3}\right\},
$$

and where $a$ and $b$ are $C^{\infty}$ in $x$ with values in the usual pseudodifferential algebra $S^{1}\left(\mathbb{R}^{2}\right)$ defined by

$$
S^{1}=\left\{q \in C^{\infty}\left(\mathbb{R}^{2}\right) /\left|\partial_{t}^{k} \partial_{\tau}^{l} q(t, \tau)\right| \leq C_{k l}\left(1+\tau^{2}\right)^{\frac{1-l}{2}}, \forall(k, l) \in \mathbb{N}^{2}\right\} .
$$

The calculations of $a$ and $b$ in (3.2) are done in the following way. The symbols of $a$ and $b$ admit expansions

$$
a(x, t, \tau)=\sum_{j \geq 0} a_{1-j}(x, t, \tau)
$$

and

$$
b(x, t, \tau)=\sum_{j \geq 0} b_{1-j}(x, t, \tau)
$$

where $a_{1-j}(x, t, \tau)$ and $b_{1-j}(x, t, \tau)$ are homogeneous of degree $1-j$ in $\tau$. The symbol of the product of pseudodifferential operators $a\left(t, x, D_{t}\right) b\left(t, x, D_{t}\right)$ is asymptotic to

$$
\sum_{k, l, n} \frac{1}{i^{n} n !} \partial_{\tau}^{n} a_{1-l}(x, t, \tau) \partial_{t}^{n} b_{1-k}(x, t, \tau) .
$$

The coefficients are then given by

$$
\left\{\begin{array}{l}
a_{1-j}+b_{1-j}=\delta_{j 1} \gamma, j \geq 0 \\
b_{1}=-i \tau \text { and } a_{1}=i \tau \\
\sum_{l+k+n=j+1} \frac{1}{i^{n} n !} \partial_{\tau}^{n} a_{1-l} \partial_{t}^{n} b_{1-k}-\frac{\partial b_{1-j}}{\partial x}=-\delta_{j 1} \alpha-\delta_{j 0} \beta i \tau, j \geq 0
\end{array}\right.
$$


with $\delta_{j k}=1$ if $j=k$ and 0 otherwise. We obtain in particular

$$
\left\{\begin{array}{l}
b_{1}=-i \tau \text { and } a_{1}=i \tau \\
b_{0}=\frac{\gamma-\beta}{2} \text { and } a_{0}=\frac{\beta+\gamma}{2} \\
b_{-1}=\frac{-\alpha+\left(\beta^{2}-\gamma^{2}\right) / 4+\left(\partial_{x}-\partial_{t}\right)(\gamma-\beta) / 2}{2 i \tau}=-a_{-1} .
\end{array}\right.
$$

(3.1) restricted to $\mathbb{R}^{+}$becomes

$$
\left\{\begin{array}{l}
\left(\partial_{t}^{2}-\partial_{x}^{2}\right) u_{e x}+\alpha(t, x) u_{e x}+\beta(t, x) \partial_{t} u_{e x}+\gamma(t, x) \partial_{x} u_{e x}=0 \text { in } \mathbb{R}^{+} \times \mathbb{R}^{+} \\
u_{e x}=0, \partial_{t} u_{e x}=0 \text { at } t=0
\end{array}\right.
$$

which together with (3.2) and Theorems 1 and 2 of [14 implies

$$
\left.\left(\partial_{x}-b\left(0, t, D_{t}\right)\right) u_{e x}\right|_{x=0} \in C^{\infty} .
$$

B. Engquist and A. Majda propose to take $B=b\left(0, t, D_{t}\right)$, although (3.6) is weaker than $\left.\left(\partial_{x}-B\right) u_{e x}\right|_{x=0}=0$. In fact, we do not know how to improve (3.6), and the numerical results obtained with this choice are good.

$B$ has an infinite expansion which is not manageable for numerical simulations. The strategy of the absorbing boundary conditions consists in truncating this expansion after a finite number of terms. For an integer $k$, the absorbing boundary condition of order $k$ is

$$
\left.\left(\partial_{x}-\sum_{j=0}^{k} b_{1-j}\left(0, t, D_{t}\right)\right) u\right|_{x=0}=0,
$$

and we approximate the solution of (3.1) by the solution $u$ of

$$
\left\{\begin{array}{l}
\left(\partial_{t}^{2}-\partial_{x}^{2}\right) u+\alpha(t, x) u+\beta(t, x) \partial_{t} u+\gamma(t, x) \partial_{x} u=0 \text { in } \mathbb{R}^{+} \times \mathbb{R}^{-} \\
\left.\left(\partial_{x}-\sum_{j=0}^{k} b_{1-j}\left(0, t, D_{t}\right)\right) u\right|_{x=0}=0 \\
u=u_{0}, \partial_{t} u=u_{1} \text { at } t=0 .
\end{array}\right.
$$

This method gives good results, and we note an improvement as $k$ increases (see section (7).

We now consider the nonlinear case. Let $u_{e x}$ be the solution of (2.1). As $f(0,0,0)=0$, we may rewrite $f$ as

$$
f\left(u_{1}, u_{2}, u_{3}\right)=-\alpha\left(u_{1}, u_{2}, u_{3}\right) u_{1}-\beta\left(u_{1}, u_{2}, u_{3}\right) u_{2}-\gamma\left(u_{1}, u_{2}, u_{3}\right) u_{3},
$$

and $u_{e x}$ satisfies (3.1), where $\alpha, \beta$ and $\gamma$ now depend on $u_{e x}$. A first idea is to follow the strategy of the linear case. For a function $f$ of the type $f\left(u, u_{t}, u_{x}\right)=$ $f_{1}(u)+f_{2}(u) u_{t}+f_{3}(u) u_{x}$ where $f_{j}$ is in $C^{\infty}(\mathbb{R}), 1 \leq j \leq 3$, and $f_{1}(0)=0$, we set $(\alpha, \beta, \gamma)=\left(-f_{1}(u) / u,-f_{2}(u),-f_{3}(u)\right)$. With this choice, we obtain the firstorder absorbing boundary condition (2.4) and the second-order absorbing boundary condition (2.5).

Remark. The pseudodifferential strategy is valid if the multiplication by $\alpha, \beta$ and $\gamma$ are pseudodifferential operators, i.e., if $\alpha, \beta$ and $\gamma$ are $C^{\infty}$. Thus, in the nonlinear case, this strategy is valid when $u_{e x}$ is smooth, but not when $u_{e x}$ has finite regularity. This justifies the use of the paradifferential calculus. 
A second idea is to linearize equation (2.1). Then, we set

$$
(\alpha, \beta, \gamma)=\left(-f_{1}^{\prime}(u)-f_{2}^{\prime}(u) \partial_{t} u-f_{3}^{\prime}(u) \partial_{x} u,-f_{2}(u),-f_{3}(u)\right)
$$

and we follow the strategy of the linear case. We do not write the boundary conditions obtained in this case, as they provide poorer numerical results than those given by boundary conditions (2.4) and (2.5).

\section{The paradifferential CALCUlus}

4.1. The paradifferential calculus of J. M. Bony. In nonlinear problems, we have to give a sense to the multiplication of functions having low regularity. J. M. Bony [4 proposes to replace the multiplication by a function $u(x), x \in \mathbb{R}^{d}$, by the operator $T_{u}$ defined as follows:

$$
\mathcal{F}\left(T_{u} v\right)(\xi)=(2 \pi)^{-d} \int_{\mathbb{R}^{d}} \chi(\xi-\eta, \eta) \mathcal{F} u(\xi-\eta) \mathcal{F} v(\eta) d \eta,
$$

where $\mathcal{F}$ is the Fourier transform and $\chi$ is a $C^{\infty}$ function in $\mathbb{R}^{d} \times\left(\mathbb{R}^{d} \backslash\{0\}\right)$ homogeneous of degree 0 in $(\xi, \eta)$ and satisfying

$$
\begin{cases}\chi(\xi, \eta)=1 & \text { if }|\xi| \leq \varepsilon_{1}|\eta| \\ \chi(\xi, \eta)=0 & \text { if }|\xi| \geq \varepsilon_{2}|\eta|\end{cases}
$$

with $0<\varepsilon_{1}<\varepsilon_{2}<1$.

Remark. If $\chi=1$, and if $u$ and $v$ are sufficiently smooth, (4.1) is exactly the multiplication of $v$ by $u$.

The paramultiplication satisfies the following properties:

(a) If $u$ is in $L^{\infty}\left(\mathbb{R}^{d}\right)$ and $v$ is in $H^{s}\left(\mathbb{R}^{d}\right)$, then $T_{u} v$ is in $H^{s}\left(\mathbb{R}^{d}\right)$ and $\left\|T_{u} v\right\|_{H^{s}\left(\mathbb{R}^{d}\right)}$ $\leq C_{s}\|u\|_{L^{\infty}\left(\mathbb{R}^{d}\right)}\|v\|_{H^{s}\left(\mathbb{R}^{d}\right)}$ for any real $s$ (see for instance [5]).

(b) Let $s>d / 2$ and let $u$ be in $H^{s}\left(\mathbb{R}^{d}\right)$. A change of function $\chi$ in $T_{u}$ modifies $T_{u}$ by the addition of a $s-d / 2$-regularizing operator, i.e., an operator mapping $H^{t}\left(\mathbb{R}^{d}\right)$ in $H^{t+s-d / 2}\left(\mathbb{R}^{d}\right)$ for any real $t$.

(c) The following result of J. M. Bony with the improvement of Y. Meyer [15] shows the relationship between the paramultiplication and nonlinear functions. Let $F$ be a real function in $C^{\infty}\left(\mathbb{R}^{N}\right)$ such that $F(0)=0$ and let $u_{1}, \ldots, u_{N}$ be real functions in $H^{s}\left(\mathbb{R}^{d}\right)$ with $s>\frac{d}{2}$. Then, $F\left(u_{1}, \ldots, u_{N}\right)$ is in $H^{s}\left(\mathbb{R}^{d}\right)$ and there is $r$ in $H^{2 s-\frac{d}{2}}\left(\mathbb{R}^{d}\right)$ such that

$$
F\left(u_{1}, \ldots, u_{N}\right)=\sum_{j=1}^{N} T_{\frac{\partial F}{\partial u_{j}}\left(u_{1}, \ldots, u_{N}\right)} u_{j}+r .
$$

4.2. The tangential paradifferential calculus of M. Sablé-Tougeron. For boundary problems, L. Hörmander [11] introduces for $s$ and $s^{\prime}$ real numbers and $d \geq 2$ an integer the spaces $H^{s, s^{\prime}}\left(\mathbb{R}^{d}\right)$ defined by

$$
\begin{aligned}
H^{s, s^{\prime}} & \left.\mathbb{R}^{d}\right) \\
& =\left\{u \in \mathcal{S}^{\prime}\left(\mathbb{R}^{d}\right) /\|u\|_{s, s^{\prime}}=(2 \pi)^{-d} \int\left(1+|\xi|^{2}\right)^{s}\left(1+\left|\xi^{\prime}\right|^{2}\right)^{s^{\prime}}|\mathcal{F} u(\xi)|^{2} d \xi<+\infty\right\},
\end{aligned}
$$

where $\mathcal{S}^{\prime}\left(\mathbb{R}^{d}\right)$ is the space of tempered distributions and where $\xi$ (resp. $\xi^{\prime}$ ) is the dual variable of $x$ (resp. $x^{\prime}$ ) with $x=\left(x^{\prime}, x_{d}\right)$ and $x^{\prime} \in \mathbb{R}^{d-1}$. L. Hörmander also 
introduces the space $H^{s, s^{\prime}}\left(\overline{\mathbb{R}}_{+}^{d}\right)$ as the set of functions $u$ in $\mathcal{D}^{\prime}\left(\mathbb{R}_{+}^{d}\right)$ such that there is $U$ in $H^{s, s^{\prime}}\left(\mathbb{R}^{d}\right)$ satisfying $U=u$ in $\mathbb{R}_{+}^{d}$. M. Sablé-Tougeron [17 proves

(d) Let $s$ and $s^{\prime}$ be real numbers such that

$$
s>\frac{1}{2}, s+s^{\prime}>\frac{d}{2} \text { and } s+2 s^{\prime}>\frac{1}{2},
$$

then $H^{s, s^{\prime}}$ is a multiplicative algebra.

M. Sablé-Tougeron defines a tangential paramultiplication which acts only on the variable $x^{\prime}$ :

$$
\mathcal{F}_{x^{\prime}}\left(T_{u}^{\prime} v\right)\left(\xi^{\prime}, x_{d}\right)=(2 \pi)^{-d+1} \int_{\mathbb{R}^{d-1}} \chi^{\prime}\left(\xi^{\prime}-\eta^{\prime}, \eta^{\prime}\right) \mathcal{F}_{x^{\prime}} u\left(\xi^{\prime}-\eta^{\prime}, x_{d}\right) \mathcal{F}_{x^{\prime}} v\left(\eta^{\prime}, x_{d}\right) d \eta^{\prime},
$$

where $\mathcal{F}_{x^{\prime}}$ is the Fourier transform with respect to $x^{\prime}$ and $\chi^{\prime}$ is a $C^{\infty}$ function in $\mathbb{R}^{d-1} \times\left(\mathbb{R}^{d-1} \backslash\{0\}\right)$ homogeneous of degree 0 in $\left(\xi^{\prime}, \eta^{\prime}\right)$ and satisfying

$$
\begin{cases}\chi^{\prime}\left(\xi^{\prime}, \eta^{\prime}\right)=1 & \text { if }\left|\xi^{\prime}\right| \leq \varepsilon_{1}\left|\eta^{\prime}\right| \\ \chi^{\prime}\left(\xi^{\prime}, \eta^{\prime}\right)=0 & \text { if }\left|\xi^{\prime}\right| \geq \varepsilon_{2}\left|\eta^{\prime}\right|\end{cases}
$$

where $0<\varepsilon_{1}<\varepsilon_{2}<1$. She proves the analog of (4.3):

(e) Let $F$ be a real function in $C^{\infty}\left(\overline{\mathbb{R}}_{+}^{d} \times \mathbb{R}^{N}\right)$ with compact support in $x$ and let $u_{1}, \ldots, u_{N}$ be real functions in $H^{s, s^{\prime}}\left(\overline{\mathbb{R}}_{+}^{d}\right)$ where $s$ and $s^{\prime}$ satisfy (4.4). Then $F\left(x, u_{1}, \ldots, u_{N}\right)$ is in $H^{s, s^{\prime}}\left(\overline{\mathbb{R}}_{+}^{d}\right)$, and there is $r$ in $H^{s, s^{\prime}+\rho}\left(\overline{\mathbb{R}}_{+}^{d}\right)$ such that

$$
F\left(x, u_{1}, \ldots, u_{N}\right)=\sum_{j=1}^{N} T_{\frac{\partial F}{\partial u_{j}}\left(x, u_{1}, \ldots, u_{N}\right)}^{\prime} u_{j}+r,
$$

where $\rho>0$ is defined by

$$
\begin{array}{ll}
\rho=\min \left(s-\frac{1}{2}, s+s^{\prime}-\frac{d}{2}\right) & \text { if } s^{\prime} \neq(d-1) / 2, \\
0<\rho<s-\frac{1}{2} & \text { if } s^{\prime}=(d-1) / 2 .
\end{array}
$$

Let $m^{\prime}$ be in $\mathbb{R}$, and let $s$ and $s^{\prime}$ be such that

$$
-\frac{d-1}{2} \leq s^{\prime}<\frac{d-1}{2} \text { and } s+s^{\prime}>\frac{d}{2} .
$$

To include the tangential paramultiplication and the tangential pseudodifferential operators in an algebra, she introduces the classes $\Sigma_{s, s^{\prime}}^{m^{\prime}}\left(\overline{\mathbb{R}}_{+}^{d}\right)$ of symbols $p\left(x, \xi^{\prime}\right)$ satisfying

$$
p=\sum_{j \leq s+s^{\prime}-\frac{d}{2}} p_{m^{\prime}-j},
$$

where $p_{m^{\prime}-j}$ is homogeneous in $\xi^{\prime}$ of degree $m^{\prime}-j, C^{\infty}$ in $\xi^{\prime} \neq 0$ and in $H^{s-j, s^{\prime}}\left(\overline{\mathbb{R}}_{+}^{d}\right)$ with respect to $x$. The tangential paradifferential operator $T_{p}^{\prime}$ is defined by

$$
\begin{aligned}
& \mathcal{F}_{x^{\prime}}\left(T_{p}^{\prime} v\right)\left(\xi^{\prime}, x_{d}\right) \\
& \quad=(2 \pi)^{-d} \int_{\mathbb{R}^{d-1}} \chi^{\prime}\left(\xi^{\prime}-\eta^{\prime}, \eta^{\prime}\right) \mathcal{F}_{x^{\prime}} p\left(\xi^{\prime}-\eta^{\prime}, x_{d}, \eta^{\prime}\right) \phi\left(\eta^{\prime}\right) \mathcal{F}_{x^{\prime}} v\left(\eta^{\prime}, x_{d}\right) d \eta,
\end{aligned}
$$

where $\chi^{\prime}$ is chosen as before, $\phi$ is in $C^{\infty}$ equal to 1 outside a compact and equal to 0 in the neighborhood of 0 , and $\mathcal{F}_{x^{\prime}} p$ is the Fourier transform of $p\left(x, \xi^{\prime}\right)$ with respect to $x^{\prime}$. We have the following properties: 
(f) A change of function $\chi^{\prime}$ in $T_{p}^{\prime}$ modifies $T_{p}^{\prime}$ by the addition of a continuous operator from $H^{t, t^{\prime}}\left(\overline{\mathbb{R}}_{+}^{d}\right)$ to $H^{t, t^{\prime}-m^{\prime}+s+s^{\prime}-\frac{d}{2}}\left(\overline{\mathbb{R}}_{+}^{d}\right)$ for $-\left(s-\left[s+s^{\prime}-\frac{d}{2}\right]\right)<$ $t \leq s-\left[s+s^{\prime}-\frac{d}{2}\right]$ and for all $t^{\prime}$ ([y] is the integral part of $y$ ).

(g) $T_{p}^{\prime}$ is continuous from $H^{t, t^{\prime}}\left(\overline{\mathbb{R}}_{+}^{d}\right)$ to $H^{t, t^{\prime}-m^{\prime}}\left(\overline{\mathbb{R}}_{+}^{d}\right)$ for $-\left(s-\left[s+s^{\prime}-\frac{d}{2}\right]\right)<$ $t \leq s-\left[s+s^{\prime}-\frac{d}{2}\right]$ and for all $t^{\prime}$.

(h) A linear continuous operator $A$ from $C_{0}^{\infty}\left(\mathbb{R}_{+}^{d}\right)$ to $C^{\infty}\left(\mathbb{R}_{+}^{d}\right)$ is said to be properly supported if for all compact $K$ of $\mathbb{R}_{+}^{d}$, there exists a compact $K^{\prime}$ of $\mathbb{R}_{+}^{d}$ such that

$$
\operatorname{supp} u \subset K \Rightarrow \operatorname{supp} A u \subset K^{\prime} \text { and } u=0 \text { on } K^{\prime} \Rightarrow A u=0 \text { on } K \text {. }
$$

$u \in H_{\text {loc }}^{t, t^{\prime}}\left(\overline{\mathbb{R}}_{+}^{d}\right)$ is said to be microlocally of class $\widetilde{H}^{t, \sigma^{\prime}}$ at a point $\left(x_{0}, \xi_{0}^{\prime}\right)$ of $\overline{\mathbb{R}}_{+}^{d} \times\left(\mathbb{R}^{d-1} \backslash\{0\}\right)$ if there is a properly supported tangential pseudodifferential operator $Q$ of order 0 elliptic at $\left(x_{0}, \xi_{0}^{\prime}\right)$ such that $Q u$ is in $H^{t, \sigma^{\prime}}\left(\overline{\mathbb{R}}_{+}^{d}\right)$. Let $t$ be such that $-\left(s-\left[s+s^{\prime}-\frac{d}{2}\right]\right)<t \leq s-\left[s+s^{\prime}-\frac{d}{2}\right]$ and let $u$ be in $H^{t, t^{\prime}}\left(\overline{\mathbb{R}}_{+}^{d}\right)$. If $u$ is microlocally of class $\widetilde{H}^{t, \sigma^{\prime}}$ at $\left(x_{0}, \xi_{0}^{\prime}\right)$, then $T_{p}^{\prime} u$ is microlocally of class $\widetilde{H}^{t, \min \left(t^{\prime}-m^{\prime}+\rho, \sigma^{\prime}-m^{\prime}\right)}$ at $\left(x_{0}, \xi_{0}^{\prime}\right)$, where $\rho$ is defined as in (4.7).

(i) If $p$ is in $\Sigma_{s, s^{\prime}}^{m^{\prime}}\left(\overline{\mathbb{R}}_{+}^{d}\right)$ and $q$ is in $\Sigma_{s, s^{\prime}}^{m^{\prime \prime}}\left(\overline{\mathbb{R}}_{+}^{d}\right)$, then there is $r$ bounded in $x_{d} \geq 0$ with values in the $s+s^{\prime}-\frac{d}{2}-m^{\prime}-m^{\prime \prime}$-regularizing operators such that

$$
T_{p}^{\prime} T_{q}^{\prime}=T_{p \# q}^{\prime}+r\left(x, D_{x^{\prime}}\right)
$$

where $p \# q$ is in $\Sigma_{s, s^{\prime}}^{m^{\prime}+m^{\prime \prime}}\left(\overline{\mathbb{R}}_{+}^{d}\right)$ and defined by

$$
p \# q=\sum_{j+k+\left|\alpha^{\prime}\right| \leq s+s^{\prime}-\frac{d}{2}} \frac{1}{i^{\left|\alpha^{\prime}\right|} \alpha^{\prime} !} \partial_{\xi^{\prime}}^{\alpha^{\prime}} p_{m^{\prime}-j} \partial_{x^{\prime}}^{\alpha^{\prime}} q_{m^{\prime \prime}-k} .
$$

In $\mathbb{R}^{d-1} \times\left[0, x_{d}^{1}\right]$, we consider the operator of evolution $L\left(x, D_{x}\right)=\partial_{x_{d}}-T_{p}^{\prime}$ where $p$ is in $\Sigma_{s, s^{\prime}}^{1}\left(\overline{\mathbb{R}}_{+}^{d}\right),\left(s, s^{\prime}\right)$ satisfies (4.4) with $\rho>1$ ( $\rho$ is defined by (4.7) $)$ and the principal symbol $p_{1}\left(x, \xi^{\prime}\right)$ of $p\left(x, \xi^{\prime}\right)$ is purely imaginary and $C^{2}$ in $\left(x, \xi^{\prime}\right)$. A point $\left(x_{0}, \xi_{0}^{\prime}\right)$ being fixed in $\mathbb{R}^{d-1} \times\{0\} \times\left(\mathbb{R}^{d-1} \backslash\{0\}\right)$, we define $\widetilde{\gamma}$ as the projection on $\xi_{d}=0$ of the bicharacteristic of $l(x, \xi)=\xi_{d}+i p_{1}\left(x, \xi^{\prime}\right)$ through $\left(x_{0}, \xi_{0}^{\prime},-i p_{1}\left(x_{0}, \xi_{0}^{\prime}\right)\right)$. Suppose that $x_{d}^{1}$ is small enough such that $\widetilde{\gamma}$, parametrized by $x_{d}$, is defined for $x_{d}$ in $\left[0, x_{d}^{1}\right]$. A. Alabidi [1] proves in particular the following result:

(j) Let $t$ and $\sigma$ be two real numbers such that $t \leq \sigma \leq t+\rho-1$ and let $u$ be in $H^{1, t-1}\left(\mathbb{R}_{+}^{d}\right)$. Suppose that $L u$ is microlocally of class $\widetilde{H}^{0, \sigma}$ on $\widetilde{\gamma}$ and that $u$ is microlocally of class $\widetilde{H}^{1, \sigma-1}$ at $\widetilde{\gamma}\left(x_{d}^{2}\right)$ with $0<x_{d}^{2}<x_{d}^{1}$. Then, for $0 \leq x_{d} \leq x_{d}^{1}, u\left(., x_{d}\right)$ is microlocally of class $H^{\sigma}$ at the point $\left(x^{\prime}, \xi^{\prime}\right)$ such that $\left(x^{\prime}, x_{d}, \xi^{\prime}\right)$ belongs to $\widetilde{\gamma}$.

\section{AbSORBING BOUNDARY CONDITIONS USING PARADIFFERENTIAL CALCULUS}

5.1. The transparent boundary condition. Let $s, f, u_{0}$ and $u_{1}$ satisfy the hypothesis of section 2 Let $u_{e x}$ in $H^{s}(] 0, T[\times \mathbb{R})$ be the solution of (2.1). In order to approximate $u_{e x}$, we look for a condition satisfied by $u_{e x}$ at $x=0$ of the form $\left.\left(\partial_{x}-B\right) u_{e x}\right|_{x=0}=0$, where $B\left(u_{e x}, t, D_{t}\right)$ is an operator. In view of the linear case and in particular of (3.6), we will limit ourselves to look for $B$ satisfying 
$\left.\left(\partial_{x}-B\right) u_{e x}\right|_{x=0} \in H_{\mathrm{loc}}^{s+\sigma}(] 0, T[)$, where $\sigma$ is as large as possible. In this study, we obtain $\sigma=s-3$. Without conditions on $B,\left.\left(\partial_{x}-B\right) u_{e x}\right|_{x=0}$ belongs at most to $H^{s-\frac{3}{2}}(] 0, T[)$. A judicious choice of $B$ therefore implies a $s-\frac{3}{2}$ gain in regularity.

If two operators $B$ and $B^{\prime}$ are well-suited, $R=B-B^{\prime}$ satisfies $\left.R u_{e x}\right|_{x=0} \in$ $H_{\text {loc }}^{2 s-3}(] 0, T[)$ for all $u_{0}, u_{1}$ with compact support in $\mathbb{R}^{-}$and $u_{e x}$ a solution of (2.1). Then, for all $h$ in $H^{s-\frac{1}{2}}(] 0, T[), R h$ belongs to $H_{\mathrm{loc}}^{2 s-3}(] 0, T[)$ (assuming that the map $\left.\left(u_{0}, u_{1}\right) \rightarrow u_{e x}\right|_{x=0}$ is onto $\left.H^{s-\frac{1}{2}}(] 0, T[)\right)$. Therefore, the difference of two such operators is $s-\frac{5}{2}$-regularizing. As $B$ is a paradifferential operator in our study, this has a link with points (b) and (f).

Remarks 1. Our method is valid for weak singularities $(s>2)$, and the larger $s$ is, the better are the results. On the other hand, it does not work for strong singularities (like shock waves, expansion waves,...) where the paradifferential calculus is not valid (for instance, (4.3) requires $s>\frac{d}{2}$ ).

2. In the case of linear equations, we obtain $\sigma=+\infty$ (see for instance [7] and [10]).

We extend $u_{e x}$ by 0 to $t<0$. As $u_{0}$ and $u_{1}$ have compact support in $\mathbb{R}^{-}$and $u_{e x}$ satisfies (2.1), $u_{e x}$ is in $H^{s}(]-\infty, T\left[\times \mathbb{R}_{+}\right)$. Moreover, as $f(0,0,0)=0, u_{e x}$ satisfies

$$
\left.\left(\partial_{t}^{2}-\partial_{x}^{2}\right) u_{e x}=f\left(J u_{e x}\right) \text { in }\right]-\infty, T\left[\times \mathbb{R}_{+},\right.
$$

where for a function $v$ depending on $(t, x)$, we define

$$
J v(t, x)=\left(v(t, x), \partial_{t} v(t, x), \partial_{x} v(t, x)\right) .
$$

We will look for $B$ as a tangential paradifferential operator with the symbol $b$ in $\Sigma_{s, 0}^{1}(]-\infty, T\left[\times \overline{\mathbb{R}}_{+}\right)((s, 0)$ satisfies (4.8) because $d=2$ in this study). Thus, we look for $b$ such that

$$
\left.\left(\partial_{x}-T_{b}^{\prime}\right) u_{e x}\right|_{x=0} \in H^{2 s-3}(]-\infty, T[) .
$$

As $u_{e x}$ is in $H^{s, 0}(]-\infty, T\left[\times \overline{\mathbb{R}}_{+}\right),(s-1,0)$ satisfies (4.4), and as $f$ is in $C^{\infty}\left(\mathbb{R}^{3}, \mathbb{R}\right)$, (e) implies

$$
f\left(J u_{e x}\right)-T_{\frac{\partial f}{\partial u_{1}}}^{\prime} u_{e x}-T_{\frac{\partial f}{\partial u_{2}}}^{\prime} \partial_{t} u_{e x}-T_{\frac{\partial f}{\partial u_{3}}}^{\prime} \partial_{x} u_{e x} \in H^{s-1, s-2}(]-\infty, T\left[\times \overline{\mathbb{R}}_{+}\right) .
$$

Then, (5.1) and (5.4) yield

$$
\partial_{x}^{2} u_{e x}-\partial_{t}^{2} u_{e x}+T_{\frac{\partial f}{\partial u_{1}}}^{\prime} u_{e x}+T_{\frac{\partial f}{\partial u_{2}}}^{\prime} \partial_{t} u_{e x}+T_{\frac{\partial f}{\partial u_{3}}}^{\prime} \partial_{x} u_{e x} \in H^{0,2 s-3}(]-\infty, T\left[\times \overline{\mathbb{R}}_{+}\right) \text {. }
$$

We define $L=\partial_{x}^{2}-\partial_{t}^{2}+T_{\frac{\partial f}{\partial u_{1}}}^{\prime}+T_{\frac{\partial f}{\partial u_{2}}}^{\prime} \partial_{t}+T_{\frac{\partial f}{\partial u_{3}}}^{\prime} \partial_{x}$, where $\frac{\partial f}{\partial u_{j}}=\frac{\partial f}{\partial u_{j}}\left(J u_{e x}\right)$ for $1 \leq j \leq 3$. The following factorization is parallel to L. Nirenberg's factorization [16] for classical pseudodifferential operators:

Proposition 1. There exist $a$ and $b$ in $\Sigma_{s, 0}^{1}(]-\infty, T\left[\times \overline{\mathbb{R}}_{+}\right)$such that $\operatorname{Im}\left(a_{1}\right)$ and $\tau$ have the same sign, and

$$
\left.L=\left(\partial_{x}-T_{a}^{\prime}\right)\left(\partial_{x}-T_{b}^{\prime}\right)+r\left(t, x, D_{t}\right),(t, x) \in\right]-\infty, T\left[\times \overline{\mathbb{R}}_{+},\right.
$$

where $r\left(t, x, D_{t}\right)$ is bounded in $x_{d} \geq 0$ with values in the $s-3$-regularizing operators.

Proof. It is done by identification and induction. We first have

$$
\left(\partial_{x}-T_{a}^{\prime}\right)\left(\partial_{x}-T_{b}^{\prime}\right)=\partial_{x}^{2}-\left(T_{a}^{\prime}+T_{b}^{\prime}\right) \partial_{x}+T_{a}^{\prime} T_{b}^{\prime}-T_{\frac{\partial b}{\partial x}}^{\prime} .
$$


Thus, $L=\left(\partial_{x}-T_{a}^{\prime}\right)\left(\partial_{x}-T_{b}^{\prime}\right)+r\left(t, x, D_{t}\right)$ is equivalent to

$$
\left\{\begin{array}{l}
T_{a}^{\prime}+T_{b}^{\prime}=-T_{\frac{\partial f}{\partial u_{3}}}^{\prime}, \\
T_{a}^{\prime} T_{b}^{\prime}-T_{\frac{\partial b}{\partial x}}^{\prime}+r\left(t, x, D_{t}\right)=-\partial_{t}^{2}+T_{\frac{\partial f}{\partial u_{1}}}^{\prime}+T_{\frac{\partial f}{\partial u_{2}}}^{\prime} \partial_{t} .
\end{array}\right.
$$

As $(s, 0)$ satisfies (4.8), (i) implies that (5.7) is equivalent to

$$
\left\{\begin{array}{l}
a_{1-j}+b_{1-j}=-\delta_{j 1} \frac{\partial f}{\partial u_{3}}, 0 \leq j \leq s-1, \\
a_{1} b_{1}=\tau^{2} \\
\sum_{l+k+n=j+1} \frac{1}{i^{n} n !} \partial_{\tau}^{n} a_{1-l} \partial_{t}^{n} b_{1-k}-\frac{\partial b_{1-j}}{\partial x}=\delta_{j 1} \frac{\partial f}{\partial u_{1}}+\delta_{j 0} \frac{\partial f}{\partial u_{2}} i \tau, 0 \leq j \leq s-2 .
\end{array}\right.
$$

The two first equalities in (5.8) and the fact that $\operatorname{Im}\left(a_{1}\right)$ and $\tau$ have the same sign yield $a_{1}=i \tau$ and $b_{1}=-i \tau$. Suppose we have found $a_{1-k}$ and $b_{1-k}$ homogeneous of degree $1-k$ in $\tau$ and in $H^{s-k, 0}(]-\infty, T\left[\times \overline{\mathbb{R}}_{+}\right)$with respect to $(t, x)$ for $0 \leq k \leq j$ and $j \leq s-2$ satisfying (5.8). Then $j+1 \leq s-1$ and the first and the third equality of (5.8) yield

$$
\begin{aligned}
2 i \tau b_{-j}= & -\sum_{l+k+n=j+1, k, l \leq j} \frac{1}{i^{n} n !} \partial_{\tau}^{n} a_{1-l} \partial_{t}^{n} b_{1-k} \\
& +\frac{\partial b_{1-j}}{\partial x}+\delta_{j 1} \frac{\partial f}{\partial u_{1}}+\delta_{j 0} \frac{\partial f}{\partial u_{2}} i \tau-\delta_{j 0} \frac{\partial f}{\partial u_{3}} i \tau .
\end{aligned}
$$

The induction hypothesis implies that $\frac{\partial b_{1-j}}{\partial x}$ is homogeneous of degree $1-j$ in $\tau$ and in $H^{s-j-1,0}(]-\infty, T\left[\times \overline{\mathbb{R}}_{+}\right)$with respect to $(t, x)$. Moreover, as $u_{e x}$ is in $H^{s, 0}(]-\infty, T\left[\times \overline{\mathbb{R}}_{+}\right)$and $(s-1,0)$ satisfies (4.4), (e) yields

$$
\delta_{j 1} \frac{\partial f}{\partial u_{1}}+\delta_{j 0} \frac{\partial f}{\partial u_{2}} i \tau-\delta_{j 0} \frac{\partial f}{\partial u_{3}} i \tau \text { is homogeneous of degree } 1-j \text { in } \tau
$$
and in $H^{s-j-1,0}(]-\infty, T\left[\times \overline{\mathbb{R}}_{+}\right)$with respect to $(t, x)$.

If $j<s-2, H^{s-j-1,0}(]-\infty, T\left[\times \overline{\mathbb{R}}_{+}\right)$is an algebra by (d), thus the induction hypothesis implies

$$
\begin{aligned}
& \sum_{l+k+n=j+1, k, l \leq j} \frac{1}{i^{n} n !} \partial_{\tau}^{n} a_{1-l} \partial_{t}^{n} b_{1-k} \text { is homogeneous of degree } 1-j \\
& \text { in } \tau \text { and in } H^{s-j-1,0}(]-\infty, T\left[\times \overline{\mathbb{R}}_{+}\right) \text {with respect to }(t, x) .
\end{aligned}
$$

If $j=s-2, H^{s-j-1,0}(]-\infty, T\left[\times \overline{\mathbb{R}}_{+}\right)=H^{1,0}(]-\infty, T\left[\times \overline{\mathbb{R}}_{+}\right)$is not an algebra, but the multiplication is continuous from $H^{t, 0}(]-\infty, T\left[\times \overline{\mathbb{R}}_{+}\right) \times H^{1,0}(]-\infty, T\left[\times \overline{\mathbb{R}}_{+}\right)$into $H^{1,0}(]-\infty, T\left[\times \overline{\mathbb{R}}_{+}\right)$for all $t>1$. In fact, by the definition of $H^{t, t^{\prime}}(]-\infty, T\left[\times \overline{\mathbb{R}}_{+}\right)$, it suffices to show that the multiplication is continuous from $H^{t}\left(\mathbb{R}^{2}\right) \times H^{1}\left(\mathbb{R}^{2}\right)$ into $H^{1}\left(\mathbb{R}^{2}\right)$ for all $t>1$, which follows from the results in $[5$. Thus, the induction hypothesis implies (5.10).

Therefore, $b_{-j}$ is homogeneous of degree $-j$ in $\tau$ and in $H^{s-j-1,0}(]-\infty, T\left[\times \overline{\mathbb{R}}_{+}\right)$ with respect to $(t, x)$ by (5.9). The first equality of (5.8) implies that $a_{-j}$ satisfies the same properties as $b_{-j}$, which concludes the proposition by induction.

The following theorem allows us to compute the transparent boundary condition (5.3): 
Theorem 2. Let $s>2$, let $u_{0}$ be in $H^{s}(\mathbb{R})$ and $u_{1}$ in $H^{s-1}(\mathbb{R})$ with compact support in $\mathbb{R}^{-}$and let $u_{e x}$ be the solution of (2.1). Let $b$ in $\Sigma_{s, 0}^{1}(]-\infty, T\left[\times \overline{\mathbb{R}}_{+}\right)$be given by $b_{1}=-i \tau$ and (5.8). Then

$$
\left.\left(\partial_{x}-T_{b}^{\prime}\right) u_{e x}\right|_{x=0} \in H^{2 s-3}(]-\infty, T[) .
$$

Proof. We define $w=\left(\partial_{x}-T_{b}^{\prime}\right) u_{e x}$, and we prove that $\left.w\right|_{x=0}$ is in $H_{\mathrm{loc}}^{2 s-3}(]-\infty, T[)$. It suffices to show that $\left.w\right|_{x=0}$ is microlocally of class $H^{2 s-3}$ with respect to $(t, \tau)$ for any $t$ in $]-\infty, T[$ and for all $\tau \neq 0$.

$u_{e x}$ is in $H^{s}(]-\infty, T\left[\times \mathbb{R}_{+}\right)$and thus in $H^{1, s-1}(]-\infty, T\left[\times \overline{\mathbb{R}}_{+}\right)$. As $b$ is in $\Sigma_{s, 0}^{1}(]-\infty, T\left[\times \overline{\mathbb{R}}_{+}\right)$and $-(s-[s-1])<1 \leq s-[s-1], T_{b}^{\prime} u_{e x}$ is in

$$
H^{1, s-2}(]-\infty, T\left[\times \overline{\mathbb{R}}_{+}\right)
$$

by (g). Moreover, $\partial_{x} u_{e x}$ belongs to $H^{1, s-2}(]-\infty, T\left[\times \overline{\mathbb{R}}_{+}\right)$because $u_{e x}$ is in $H^{s}(]-\infty, T\left[\times \mathbb{R}_{+}\right)$and thus in $H^{2, s-2}(]-\infty, T\left[\times \overline{\mathbb{R}}_{+}\right)$as $s-2>0$. Therefore, $w$ belongs to $H^{1, s-2}(]-\infty, T\left[\times \overline{\mathbb{R}}_{+}\right)$. The factorization of Proposition 1 yields

$$
\left(\partial_{x}-T_{a}^{\prime}\right) w=L u_{e x}-r\left(t, x, D_{t}\right) u_{e x}
$$

$u_{e x}$ is in $H^{0, s}(]-\infty, T\left[\times \overline{\mathbb{R}}_{+}\right)$and $r\left(t, x, D_{t}\right)$ is bounded in $x_{d} \geq 0$ with values in the $s-3$-regularizing operators. Therefore, $r\left(t, x, D_{t}\right) u_{e x}$ belongs to

$$
H^{0,2 s-3}(]-\infty, T\left[\times \overline{\mathbb{R}}_{+}\right) \text {. }
$$

(5.5) and (5.12) yield

$$
\left(\partial_{x}-T_{a}^{\prime}\right) w \in H^{0,2 s-3}(]-\infty, T\left[\times \overline{\mathbb{R}}_{+}\right) .
$$

Let $\left(t_{0}, \tau_{0}\right)$ be such that $\left.t_{0} \in\right]-\infty, T\left[\right.$ and $\tau_{0} \neq 0$. The bicharacteristic of $\xi+i a_{1}=\xi-\tau$ through $\left(t_{0}, x=0, \tau_{0}\right)$ satisfies $x+t=t_{0}$ and $\tau=\tau_{0}$. Therefore, there exists $\left(t_{1}, x_{1}\right)$ in $]-\infty, T\left[\times \overline{\mathbb{R}}_{+}\right.$on this bicharacteristic such that $t_{1}<0$. As $t_{1}<0$ and $u_{e x}=0$ for $t<0, u_{e x}$ is microlocally of class $\widetilde{H}^{1,+\infty}$ in $\left(t_{1}, x_{1}, \tau_{0}\right)$. As $-(s-[s-1])<1 \leq s-[s-1], T_{b}^{\prime} u_{e x}$ is microlocally of class $\widetilde{H}^{1,2 s-3}$ in $\left(t_{1}, x_{1}, \tau_{0}\right)$ by (h). As $s>2, \rho=s-1>1$, and (5.13) and (j) imply that $\left.w\right|_{x=0}$ is microlocally of class $H^{2 s-3}$ in $\left(t_{0}, \tau_{0}\right)$ (we can use (j) with $t=s-1$ and $\sigma=2 s-2$ for instance). Therefore, $\left.w\right|_{x=0}$ is microlocally of class $H^{2 s-3}$ with respect to $(t, \tau)$ for all $t$ in ]$-\infty, T[$ and for all $\tau \neq 0$. This implies

$$
\left.\left(\partial_{x}-T_{b}^{\prime}\right) u_{e x}\right|_{x=0} \in H_{\text {loc }}^{2 s-3}(]-\infty, T[) .
$$

Thanks to (5.8), we can compute the symbol of $b$. In particular, we obtain

$$
\begin{aligned}
b_{1}= & -i \tau \\
b_{0}= & \frac{1}{2}\left(\frac{\partial f}{\partial u_{2}}-\frac{\partial f}{\partial u_{3}}\right), \\
b_{-1}= & \frac{1}{4 i \tau}\left(\partial_{t}\left(\frac{\partial f}{\partial u_{3}}\right)+\partial_{x}\left(\frac{\partial f}{\partial u_{2}}\right)-\partial_{t}\left(\frac{\partial f}{\partial u_{2}}\right)-\partial_{x}\left(\frac{\partial f}{\partial u_{3}}\right)\right) \\
& +\frac{1}{8 i \tau}\left(\left(\frac{\partial f}{\partial u_{2}}\right)^{2}-\left(\frac{\partial f}{\partial u_{3}}\right)^{2}\right)+\frac{1}{2 i \tau} \frac{\partial f}{\partial u_{1}} .
\end{aligned}
$$

5.2. The absorbing boundary conditions. Theorem 2 implies the following corollary.

Corollary 1. Let $s>2$, let $u_{0}$ be in $H^{s}(\mathbb{R})$ and $u_{1}$ be in $H^{s-1}(\mathbb{R})$ with compact support in $\mathbb{R}^{-}$, and let $u_{e x}$ be the solution of (2.1). Let $b$ in $\Sigma_{s, 0}^{1}(]-\infty, T\left[\times \overline{\mathbb{R}}_{+}\right)$be given by $b_{1}=-i \tau$ and (5.8). For $0 \leq k \leq s-2$, we define $b^{k}=\sum_{j=0}^{k} b_{1-j}$. Then

$$
\left.\left(\partial_{x}-T_{b^{k}}^{\prime}\right) u_{e x}\right|_{x=0} \in H^{\min (s+k-1 / 2,2 s-3)}(]-\infty, T[) .
$$


Proof. $b-b^{k}=\sum_{k<j<s-1} b_{1-j}$, thus $b-b^{k}$ is in $\Sigma_{s-k, 0}^{-k}(]-\infty, T\left[\times \overline{\mathbb{R}}_{+}\right)$. As $u_{e x}$ is in $H^{s}(]-\infty, T[\times \mathbb{R}), u_{e x}$ is in $H^{1, s-1}(]-\infty, T\left[\times \overline{\mathbb{R}}_{+}\right)$, and $(\mathrm{g})$ implies that $T_{b-b^{k}}^{\prime} u_{e x}$ belongs to $H^{1, s-1+k}(]-\infty, T\left[\times \overline{\mathbb{R}}_{+}\right)$. Taking the trace at $x=0$, we obtain

$$
\left.T_{b-b^{k}}^{\prime} u_{e x}\right|_{x=0} \in H^{s+k-1 / 2}(]-\infty, T[) .
$$

As $\left.\left(\partial_{x}-T_{b^{k}}^{\prime}\right) u_{e x}\right|_{x=0}=\left.\left(\partial_{x}-T_{b}^{\prime}\right) u_{e x}\right|_{x=0}-\left.T_{b^{k}-b}^{\prime} u_{e x}\right|_{x=0}$, (5.11) and (5.17) imply (5.16).

Remark. $\left.T_{b^{k}}^{\prime} u_{e x}\right|_{x=0}=\left.T_{b^{k}} u_{e x}\right|_{x=0}$, where $T$ is the one-dimensional paramultiplication of J. M. Bony.

Corollary 1 motivates the following definition:

Definition 1. Let $k$ be a positive integer. The absorbing boundary condition of order $k$ is

$$
\left.\left(\partial_{x}-T_{b^{k}}\right) u\right|_{x=0}=0 .
$$

The absorbing boundary condition of order 0 is

$$
\partial_{x} u+\partial_{t} u=0 .
$$

For $k \geq 1,\left.T_{b^{k}} u\right|_{x=0}$ is a paradifferential operator. In order to simplify the numerical computations, we prefer to replace this operator in (5.18) with other nonlinear operators, using (c). Thus, we look for a boundary condition of the following type:

$$
\left.\left(\partial_{t}^{l_{k}} \partial_{x} u+\partial_{t}^{l_{k}+1} u+f_{k}\left(J_{l_{k}} u\right)\right)\right|_{x=0}=0,
$$

where $l_{k} \geq k-1, f_{k}$ is a $C^{\infty}$ function such that $f_{k}(0)=0, J_{l_{k}} u=\left(\partial_{t}^{l} u, 0 \leq l \leq\right.$ $\left.l_{k}, \partial_{t}^{j} \partial_{x} u, 0 \leq j \leq l_{k}-1\right)$, and satisfying

$$
\left.\left(\partial_{t}^{l_{k}} \partial_{x} u_{e x}+\partial_{t}^{l_{k}+1} u_{e x}+f_{k}\left(J_{l_{k}} u_{e x}\right)\right)\right|_{x=0} \in H_{\mathrm{loc}}^{\min \left(s+k-l_{k}-1 / 2,2 s-3-l_{k}\right)}(]-\infty, T[) .
$$

When such a function $f_{k}$ exists, we call (5.20) an absorbing boundary condition of order $k$, and we prefer it to (5.18). We give the first absorbing boundary condition in the general case, and the second absorbing boundary condition for a nonlinearity $f$ of the type $f\left(u, u_{t}, u_{x}\right)=f_{1}(u)+f_{2}(u) u_{t}+f_{3}(u) u_{x}$, where $f_{j}$ is in $C^{\infty}(\mathbb{R})$, $1 \leq j \leq 3$, and $f_{1}(0)=0$.

5.2.1. The first-order absorbing boundary condition. When $k=1$ and $s \geq 3$, (5.16) becomes

$$
\left.\left(\partial_{x}+\partial_{t}-T_{b_{0}}\right) u_{e x}\right|_{x=0} \in H^{\min (s+1 / 2,2 s-3)}(]-\infty, T[) .
$$

Differentiating in time and using the fact that $\left.T_{\partial_{t} b_{0}} u_{e x}\right|_{x=0}$ is in $H^{s-1 / 2}(]-\infty, T[)$ by (a), we obtain

$$
\left.\left(\left(\partial_{t} \partial_{x}+\partial_{t}^{2}\right) u_{e x}-T_{b_{0}} \partial_{t} u_{e x}\right)\right|_{x=0} \in H^{\min (s-1 / 2,2 s-4)}(]-\infty, T[) .
$$

Using that $\left.\left(\partial_{t}+\partial_{x}\right) u_{e x}\right|_{x=0} \in H^{\min (s-1 / 2,2 s-4)}(]-\infty, T[)$ by (5.16), we have

$$
\begin{aligned}
& \left.T_{b_{0}} \partial_{t} u_{e x}\right|_{x=0}=1 / 2\left(\left.T_{\frac{\partial f}{\partial u_{2}}} \partial_{t} u_{e x}\right|_{x=0}-\left.T_{\frac{\partial f}{\partial u_{3}}} \partial_{t} u_{e x}\right|_{x=0}\right) \\
& =1 / 2\left(\left.T_{\frac{\partial f}{\partial u_{2}}} \partial_{t} u_{e x}\right|_{x=0}+\left.T_{\frac{\partial f}{\partial u_{3}}} \partial_{x} u_{e x}\right|_{x=0}\right)+H^{\min (s-1 / 2,2 s-4)}(]-\infty, T[) .
\end{aligned}
$$

As

$$
\left.\left(f\left(J u_{e x}\right)-T_{\frac{\partial f}{\partial u_{1}}} u_{e x}-T_{\frac{\partial f}{\partial u_{2}}} \partial_{t} u_{e x}-T_{\frac{\partial f}{\partial u_{3}}} \partial_{x} u_{e x}\right)\right|_{x=0} \in H^{2 s-7 / 2}(]-\infty, T[)
$$


by (c) and as $\left.T_{\frac{\partial f}{\partial u_{1}}} u_{e x}\right|_{x=0} \in H^{s-1 / 2}(]-\infty, T[$ ) by (a), we get

$$
\left.T_{b_{0}} \partial_{t} u_{e x}\right|_{x=0}=1 /\left.2 f\left(u_{e x}, \partial_{t} u_{e x}, \partial_{x} u_{e x}\right)\right|_{x=0}+H^{\min (s-1 / 2,2 s-4)}(]-\infty, T[) .
$$

(5.23) and (5.25) yield

(5.26) $\left.\left(\left(\partial_{t} \partial_{x}+\partial_{t}^{2}\right) u_{e x}-1 / 2 f\left(u_{e x}, \partial_{t} u_{e x}, \partial_{x} u_{e x}\right)\right)\right|_{x=0} \in H^{\min (s-1 / 2,2 s-4)}(]-\infty, T[)$, which by (5.20) and (5.21) implies

Proposition 2. We have the first-order absorbing boundary condition

$$
\left.\left(\partial_{t} \partial_{x} u+\partial_{t}^{2} u-f\left(u, \partial_{t} u, \partial_{x} u\right) / 2\right)\right|_{x=0}=0 .
$$

5.2.2. The case $f\left(u, u_{t}, u_{x}\right)=f_{1}(u)+f_{2}(u) u_{t}+f_{3}(u) u_{x}$. For $f\left(u, u_{t}, u_{x}\right)=f_{1}(u)+$ $f_{2}(u) u_{t}+f_{3}(u) u_{x}$, (5.15) implies $b_{0}=\left(f_{2}(u)-f_{3}(u)\right) / 2$ and $b_{-1}=f_{1}^{\prime}(u) /(2 i \tau)+$ $\left(f_{2}^{\prime}(u)+f_{3}^{\prime}(u)\right)\left(\partial_{x} u+\partial_{t} u\right) /(4 i \tau)+\left(f_{2}^{2}(u)-f_{3}^{2}(u)\right) /(8 i \tau)$. When $k=1$ and $s \geq 3$, (5.16) becomes

$$
\left.\left(\partial_{x}+\partial_{t}-1 / 2 T_{f_{2}\left(u_{e x}\right)-f_{3}\left(u_{e x}\right)}\right) u_{e x}\right|_{x=0} \in H^{\min (s+1 / 2,2 s-3)}(]-\infty, T[) .
$$

$\left.u_{e x}\right|_{x=0}$ is in $H^{s-1 / 2}(]-\infty, T[)$, so that $\left.\left(T_{f_{2}\left(u_{e x}\right)-f_{3}\left(u_{e x}\right)} u_{e x}-F\left(u_{e x}\right)\right)\right|_{x=0}$ is in $H^{2 s-3 / 2}(]-\infty, T[)$ by (c), where $F$ is a primitive of $f_{2}-f_{3}$ vanishing at 0 . (5.28) becomes

$$
\left.\left(\partial_{x} u_{e x}+\partial_{t} u_{e x}-F\left(u_{e x}\right) / 2\right)\right|_{x=0} \in H^{\min (s+1 / 2,2 s-3)}(]-\infty, T[),
$$

which by (5.20) and (5.21) implies

Proposition 3. When $f\left(u, u_{t}, u_{x}\right)=f_{1}(u)+f_{2}(u) u_{t}+f_{3}(u) u_{x}$, we have the firstorder absorbing boundary condition

$$
\left.\left(\partial_{x} u+\partial_{t} u-F(u) / 2\right)\right|_{x=0}=0,
$$

where $F$ is the primitive of $f_{2}-f_{3}$ vanishing at 0 .

When $k=2$ and $s \geq 4$, (5.16) becomes

$$
\left.\left(\left(\partial_{x}+\partial_{t}-1 / 2 T_{f_{2}\left(u_{e x}\right)-f_{3}\left(u_{e x}\right)}\right) u_{e x}-T_{b_{-1}} u_{e x}\right)\right|_{x=0} \in H^{\min (s+3 / 2,2 s-3)}(]-\infty, T[) .
$$

Using the fact that $\left.\left(T_{f_{2}\left(u_{e x}\right)-f_{3}\left(u_{e x}\right)} u_{e x}-F\left(u_{e x}\right)\right)\right|_{x=0}$ is in $H^{2 s-3 / 2}(]-\infty, T[)$ by (c) and $\left.T_{\partial_{t} b_{-1}} u_{e x}\right|_{x=0}$ is in $H^{s+1 / 2}(]-\infty, T[)$ by (a), and differentiating in time, we obtain

$$
\begin{aligned}
\left(\partial_{t} \partial_{x} u_{e x}+\partial_{t}^{2} u_{e x}+1 / 2\left(f_{3}\left(u_{e x}\right)\right.\right. & \left.\left.-f_{2}\left(u_{e x}\right)\right) \partial_{t} u_{e x}-T_{b_{-1}} \partial_{t} u_{e x}\right)\left.\right|_{x=0} \\
& \in H^{\min (s+1 / 2,2 s-4)}(]-\infty, T[) .
\end{aligned}
$$

By (c) we have

$$
\begin{aligned}
T_{b_{-1}} \partial_{t} u_{e x}=1 / 2 T_{f_{1}^{\prime}(u)} & u_{e x}+1 / 4 T_{\left(f_{2}^{\prime}\left(u_{e x}\right)+f_{3}^{\prime}\left(u_{e x}\right)\right)\left(\partial_{x} u_{e x}+\partial_{t} u_{e x}\right)} u_{e x} \\
& +1 / 8 T_{\left(f_{2}\left(u_{e x}\right)\right)^{2}-\left(f_{3}\left(u_{e x}\right)\right)^{2} u_{e x}} \\
=f_{1}\left(u_{e x}\right) / 2 & +\left(f_{3}\left(u_{e x}\right)+f_{2}\left(u_{e x}\right)\right)\left(\partial_{x} u_{e x}+\partial_{t} u_{e x}\right) / 4 \\
& -1 / 4 T_{f_{2}\left(u_{e x}\right)+f_{3}\left(u_{e x}\right)}\left(\partial_{x} u_{e x}+\partial_{t} u_{e x}\right)+1 / 8 F_{1}\left(u_{e x}\right) \\
& +H^{2 s-7 / 2}(]-\infty, T[),
\end{aligned}
$$

where $F_{1}$ is a primitive of $f_{2}^{2}-f_{3}^{2}$ vanishing at 0 . (5.29) yields

$$
\begin{aligned}
T_{f_{2}\left(u_{e x}\right)+f_{3}\left(u_{e x}\right)}\left(\partial_{x} u_{e x}+\partial_{t} u_{e x}\right)=1 / 2 T_{f_{2}\left(u_{e x}\right)+f_{3}\left(u_{e x}\right)} F\left(u_{e x}\right) & \\
& +H^{\min (s+1 / 2,2 s-3)}(]-\infty, T[),
\end{aligned}
$$


and using (c) implies

$$
T_{f_{2}\left(u_{e x}\right)+f_{3}\left(u_{e x}\right)} F\left(u_{e x}\right)=-F_{1}\left(u_{e x}\right)+H^{s+1 / 2}(]-\infty, T[) .
$$

(5.32), (5.33), (5.34) and (5.35) yield

$$
\begin{aligned}
\left(\partial_{t} \partial_{x} u_{e x}\right. & +\partial_{t}^{2} u_{e x}-1 / 2 f_{1}\left(u_{e x}\right) \\
& \left.+1 / 4\left(f_{3}\left(u_{e x}\right)-3 f_{2}\left(u_{e x}\right)\right) \partial_{t} u_{e x}-1 / 4\left(f_{2}\left(u_{e x}\right)+f_{3}\left(u_{e x}\right)\right) \partial_{x} u_{e x}\right)\left.\right|_{x=0} \\
& \quad H^{\min (s+1 / 2,2 s-4)}(]-\infty, T[),
\end{aligned}
$$

and using (5.20) and (5.21) we get

Proposition 4. When $f\left(u, u_{t}, u_{x}\right)=f_{1}(u)+f_{2}(u) u_{t}+f_{3}(u) u_{x}$, we have the secondorder absorbing boundary condition

$\left.\left(\partial_{t} \partial_{x} u+\partial_{t}^{2} u-1 / 2 f_{1}(u)+1 / 4\left(f_{3}(u)-3 f_{2}(u)\right) \partial_{t} u-1 / 4\left(f_{2}(u)+f_{3}(u)\right) \partial_{x} u\right)\right|_{x=0}=0$.

Remarks 1. Proposition 1 is similar to Nirenberg's pseudodifferential factorization [16] used in [7]. However, paralinearizing (5.1) in (5.5) and replacing paradifferential operators by local operators (section 5.2) are specific to our strategy. In particular, it is different from the strategy consisting in the linearization of $f\left(u_{e x}, \partial_{t} u_{e x}, \partial_{x} u_{e x}\right)$ (see the end of section 3 for an explanation of this strategy). In fact, the operators $T_{\partial_{u_{j}} f}^{\prime}, 1 \leq j \leq 3$, appearing in the paralinearization of (5.1) in (5.5) are quite different from the multiplication by $\partial_{u_{j}} f, 1 \leq j \leq 3$, which comes from the linearization of (5.1).

2. In the linear case, our strategy gives the same absorbing boundary conditions as those obtained with the pseudodifferential calculus.

\section{Existence AND UniQuenEss RESUlts FOR THE NONLINEAR APPROXIMATE PROBLEMS}

In this section, we prove Theorem [1. The proof contains two steps:

- First step: We show the existence and uniqueness of a $u$ solution of a linear nonhomogeneous problem in sufficiently regular spaces so that $f(J u)$ and $f_{1}(J u)$ are well defined, where $J$ is defined by (5.2).

- Second step: We define an iterative scheme where $u_{l+1}$ is the solution of the linear problem of the previous step having $f\left(J u_{l}\right)$ as the right-hand side and where $f_{1}$ is estimated at $u_{l}$. We use the first step to show that the scheme is well defined. The convergence for sufficiently small $T$ is obtained through energy estimates.

6.1. The linear nonhomogeneous problem. We study the following linear nonhomogeneous problem:

$$
\left\{\begin{array}{l}
\left.\left(\partial_{t}^{2}-\partial_{x}^{2}\right) u+u=g \text { in }\right] 0, T\left[\times \mathbb{R}^{-}\right. \\
\left(\partial_{x}+\partial_{t}\right) u=h \text { at } x=0 \\
u=u_{0}, \partial_{t} u=u_{1} \text { at } t=0 \text { in } \mathbb{R}^{-} .
\end{array}\right.
$$

We have a result of existence, uniqueness and regularity for the weak formulation: 
Proposition 5. Let $u_{0}$ be in $H^{2}\left(\mathbb{R}^{-}\right)$and $u_{1}$ in $H^{1}\left(\mathbb{R}^{-}\right)$with support in $\mathbb{R}^{-}$. Let $g$ be in $H^{1}(] 0, T\left[\times \mathbb{R}^{-}\right)$and let $h$ in $H^{1}(] 0, T[)$ be such that $h(0)=0$. Then, there exists a unique solution $u$ with $\partial^{\alpha} u$ in $L^{\infty}(] 0, T\left[, L^{2}\left(\mathbb{R}^{-}\right)\right)$for all $|\alpha| \leq 2$ of the following weak formulation:

$$
\left\{\begin{array}{l}
\forall v \in H^{1}\left(\mathbb{R}^{-}\right): \\
\frac{d^{2}}{d t^{2}}(u, v)+\left(\partial_{x} u, \partial_{x} v\right)+(u, v)+\frac{d}{d t} u(., 0) v(0) \\
=h v(0)+(g, v) \text { in } \mathcal{D}^{\prime}(0, T) .
\end{array}\right.
$$

Moreover

$$
\begin{aligned}
\max _{|\alpha| \leq 2} & \left\|\partial^{\alpha} u\right\|_{L^{\infty}(] 0, T\left[, L^{2}\left(\mathbb{R}^{-}\right)\right)}^{2} \\
\leq C e^{T}\left(\|g\|_{H^{1}(] 0, T\left[\times \mathbb{R}^{-}\right)}^{2}+\|h\|_{H^{1}(] 0, T[)}^{2}\right. & \\
& \left.+\left\|u_{0}\right\|_{H^{2}\left(\mathbb{R}^{-}\right)}^{2}+\left\|u_{1}\right\|_{H^{1}\left(\mathbb{R}^{-}\right)}^{2}+\|g(0, .)\|_{L^{2}\left(\mathbb{R}^{-}\right)}^{2}\right)
\end{aligned}
$$

where $C$ is a universal constant.

Proof. Multiplying the equation by $\partial_{t} u$ and integrating it in space yields

$$
\begin{aligned}
& \frac{1}{2} \frac{d}{d t}\left(\left\|\partial_{t} u(t, .)\right\|_{L^{2}\left(\mathbb{R}^{-}\right)}^{2}+\|u(t, .)\|_{H^{1}\left(\mathbb{R}^{-}\right)}^{2}\right)+\partial_{t} u(t, 0)^{2} \\
& =\left(g(t, .), \partial_{t} u(t, .)\right)+h(t) \partial_{t} u(t, 0) .
\end{aligned}
$$

Using Cauchy-Schwartz's Lemma, the inequality $a b \leq a^{2} / 2+b^{2} / 2$, and Gronwall's Lemma yields

$$
\begin{aligned}
& \left\|\partial_{t} u(t, .)\right\|_{L^{2}\left(\mathbb{R}^{-}\right)}^{2}+\|u(t, .)\|_{H^{1}\left(\mathbb{R}^{-}\right)}^{2} \\
& \leq e^{T}\left(\left\|u_{1}\right\|_{L^{2}\left(\mathbb{R}^{-}\right)}^{2}+\left\|u_{0}\right\|_{H^{1}\left(\mathbb{R}^{-}\right)}^{2}+\|g\|_{L^{2}(] 0, T\left[\times \mathbb{R}^{-}\right)}^{2}+\|h\|_{L^{2}(] 0, T[)}^{2}\right) .
\end{aligned}
$$

(6.4) implies the uniqueness in $H^{2}(] 0, T\left[\times \mathbb{R}^{-}\right)$when $g$ is in $L^{2}(] 0, T\left[\times \mathbb{R}^{-}\right)$and $h$ belongs to $L^{2}(] 0, T[)$.

We differentiate the equation in time. $w=\partial_{t} u$ is the solution of

$$
\left\{\begin{array}{l}
\left.\left(\partial_{t}^{2}-\partial_{x}^{2}\right) w+w=\partial_{t} g \text { in }\right] 0, T\left[\times \mathbb{R}^{-},\right. \\
\left(\partial_{x}+\partial_{t}\right) w=h^{\prime} \text { at } x=0, \\
w=u_{1}, \partial_{t} w=\partial_{t}^{2} u(0, .) \text { at } t=0 \text { in } \mathbb{R}^{-},
\end{array}\right.
$$

thus (6.4) implies

$$
\begin{aligned}
& \left\|\partial_{t}^{2} u\right\|_{L^{\infty}(] 0, T\left[, L^{2}\left(\mathbb{R}^{-}\right)\right)}^{2}+\left\|\partial_{t} u\right\|_{L^{\infty}(] 0, T\left[, H^{1}\left(\mathbb{R}^{-}\right)\right)}^{2} \\
& \leq e^{T}\left(\left\|\partial_{t}^{2} u(0, .)\right\|_{L^{2}\left(\mathbb{R}^{-}\right)}^{2}+\left\|u_{1}\right\|_{H^{1}\left(\mathbb{R}^{-}\right)}^{2}+\left\|\partial_{t} g\right\|_{L^{2}(] 0, T\left[\times \mathbb{R}^{-}\right)}^{2}+\left\|h^{\prime}\right\|_{L^{2}(] 0, T[)}^{2}\right) .
\end{aligned}
$$

We shall find a bound on $\left\|\partial_{t}^{2} u(0, .)\right\|_{L^{2}\left(\mathbb{R}^{-}\right)}^{2}$. Taking $v=\partial_{t}^{2} u(0,$.$) and t=0$ in weak formulation (6.1) yields

$$
\begin{aligned}
& \left\|\partial_{t}^{2} u(0, .)\right\|_{L^{2}\left(\mathbb{R}^{-}\right)}^{2}+\left(\partial_{x} u_{0}, \partial_{x} \partial_{t}^{2} u(0, .)\right)+\left(u_{0}, \partial_{t}^{2} u(0, .)\right)+u_{1}(0) \partial_{t}^{2} u(0,0) \\
& =h(0) \partial_{t}^{2} u(0,0)+\left(g(0, .), \partial_{t}^{2} u(0, .)\right)
\end{aligned}
$$

which implies after integration by parts that

$$
\begin{aligned}
\left\|\partial_{t}^{2} u(0, .)\right\|_{L^{2}\left(\mathbb{R}^{-}\right)}^{2} & \\
\leq & \left(\left\|\partial_{x}^{2} u_{0}\right\|_{L^{2}\left(\mathbb{R}^{-}\right)}+\left\|u_{0}\right\|_{L^{2}\left(\mathbb{R}^{-}\right)}+\|g(0, .)\|_{L^{2}\left(\mathbb{R}^{-}\right)}\right)\left\|\partial_{t}^{2} u(0, .)\right\|_{L^{2}\left(\mathbb{R}^{-}\right)} \\
& +\left(-\partial_{x} u_{0}(0)-u_{1}(0)+h(0)\right) \partial_{t}^{2} u(0,0) .
\end{aligned}
$$

By hypothesis, $\partial_{x} u_{0}(0)=u_{1}(0)=h(0)=0$. Thus

$$
\left\|\partial_{t}^{2} u(0, .)\right\|_{L^{2}\left(\mathbb{R}^{-}\right)} \leq\left\|\partial_{x}^{2} u_{0}\right\|_{L^{2}\left(\mathbb{R}^{-}\right)}+\left\|u_{0}\right\|_{L^{2}\left(\mathbb{R}^{-}\right)}+\|g(0, .)\|_{L^{2}\left(\mathbb{R}^{-}\right)},
$$


which together with (6.6) yields

$$
\begin{aligned}
& \left\|\partial_{t}^{2} u\right\|_{L^{\infty}(] 0, T\left[, L^{2}\left(\mathbb{R}^{-}\right)\right)}^{2}+\left\|\partial_{t} u\right\|_{L^{\infty}(] 0, T\left[, H^{1}\left(\mathbb{R}^{-}\right)\right)}^{2} \\
& \leq e^{T}\left(3\left\|u_{0}\right\|_{H^{2}\left(\mathbb{R}^{-}\right)}^{2}+\left\|u_{1}\right\|_{H^{1}\left(\mathbb{R}^{-}\right)}^{2}\right. \\
& \left.+3\|g(0, .)\|_{L^{2}\left(\mathbb{R}^{-}\right)}^{2}+\left\|\partial_{t} g\right\|_{L^{2}(] 0, T\left[\times \mathbb{R}^{-}\right)}^{2}+\left\|h^{\prime}\right\|_{L^{2}(] 0, T[)}^{2}\right) .
\end{aligned}
$$

If $u_{0}$ is in $H^{2}\left(\mathbb{R}^{-}\right)$and $u_{1}$ in $H^{1}\left(\mathbb{R}^{-}\right)$with support in $\mathbb{R}^{-}$, and if $g$ belongs to $H^{1}(] 0, T\left[, L^{2}\left(\mathbb{R}^{-}\right)\right)$and $h$ is in $H^{1}(] 0, T[)$ such that $h(0)=0$, then (6.4) and (6.7) give the existence of a solution $u$ such that $\partial^{\alpha} u$ is in $L^{\infty}\left((] 0, T\left[, L^{2}\left(\mathbb{R}^{-}\right)\right)\right)$for $|\alpha| \leq 2$ using a Galerkin method.

As $\partial_{x}^{2} u=\partial_{t}^{2} u+u-g$, we get

$$
\begin{aligned}
& \left\|\partial_{x}^{2} u\right\|_{L^{\infty}\left(\left[0, T\left[, L^{2}\left(\mathbb{R}^{-}\right)\right)\right.\right.}^{2} \leq 2\left\|\partial_{t}^{2} u\right\|_{L^{\infty}(] 0, T\left[, L^{2}\left(\mathbb{R}^{-}\right)\right)}^{2}+2\|g\|_{L^{\infty}(] 0, T\left[, L^{2}\left(\mathbb{R}^{-}\right)\right)}^{2} \\
& \leq 2\left\|\partial_{t}^{2} u\right\|_{L^{\infty}(] 0, T\left[, L^{2}\left(\mathbb{R}^{-}\right)\right)}^{2}+4\|g(0, .)\|_{L^{2}\left(\mathbb{R}^{-}\right)}^{2}+4 T\left\|\partial_{t} g\right\|_{L^{2}(] 0, T\left[, L^{2}\left(\mathbb{R}^{-}\right)\right)}^{2} .
\end{aligned}
$$

Finally, (6.4), (6.7) and (6.8) imply (6.2) with $C=10 e$.

\subsection{The iterative scheme.}

- First, we define $u_{l+1}$ from $u_{l}$ as the solution of the linear problem with right-hand sides $g=f\left(J u_{l}\right)$ and $h=\partial^{-1} f_{1}\left(J u_{l}\right)$, where $J$ is defined by (5.2).

- We prove that for sufficiently small $T>0$, the sequence $\left(\partial^{\alpha} u_{l}\right)$ is bounded in $L^{\infty}(] 0, T\left[, L^{2}\left(\mathbb{R}^{-}\right)\right)$for $|\alpha| \leq 2$.

- Finally, we show that $\left(\partial^{\alpha} u_{l}\right)$ is a Cauchy sequence in $L^{\infty}(] 0, T\left[, L^{2}\left(\mathbb{R}^{-}\right)\right)$ for $|\alpha| \leq 2$, and that the limit $u$ satisfies the nonlinear problem.

In order to define $\left(u_{l}\right)$, we need the following lemma:

Lemma 1. Let $v^{1}$ and $v^{2}$ be such that $\partial^{\alpha} v^{j}$ is in $L^{\infty}(] 0, T\left[, L^{2}\left(\mathbb{R}^{-}\right)\right)$for $|\alpha| \leq 2$ and $j=1,2$. Let $g_{j}=f\left(J v^{j}\right), f_{1}^{j}=f_{1}\left(J v^{j}\right)$ and let $h_{j}=\partial_{t}^{-1} f_{1}^{j}$ for $j=1,2$. Then, $\partial^{\alpha} g_{j}$ is in $L^{\infty}(] 0, T\left[, L^{2}\left(\mathbb{R}^{-}\right)\right)$for $|\alpha| \leq 1$ and $h_{j}$ and $h_{j}^{\prime}$ are in $L^{\infty}(] 0, T[)$. Moreover, there exists an increasing function $\theta$ such that

$$
\begin{gathered}
\max _{|\alpha| \leq 1}\left\|\partial^{\alpha}\left(g_{1}-g_{2}\right)\right\|_{L^{\infty}(] 0, T\left[, L^{2}\left(\mathbb{R}^{-}\right)\right)} \\
+\left\|h_{1}-h_{2}\right\|_{\left.\left.L^{\infty}(] 0, T\right]\right)}+\left\|h_{1}^{\prime}-h_{2}^{\prime}\right\|_{L^{\infty}(] 0, T[)} \\
\leq \sqrt{1+T^{2}} \theta\left(\max _{|\alpha| \leq 2}\left\|\partial^{\alpha} v^{1}\right\|_{L^{\infty}(] 0, T\left[L^{2}\left(\mathbb{R}^{-}\right)\right)}+\left\|\partial^{\alpha} v^{2}\right\|_{L^{\infty}(] 0, T\left[, L^{2}\left(\mathbb{R}^{-}\right)\right)}\right) \\
\max _{|\alpha| \leq 1}\left\|\partial^{\alpha}\left(v^{1}-v^{2}\right)\right\|_{L^{\infty}(] 0, T\left[L^{2}\left(\mathbb{R}^{-}\right)\right) .}
\end{gathered}
$$

Proof. As $f(0,0,0)=0$ and $f_{1}(0,0,0)=0$, 6.9) implies that $\partial^{\alpha} g_{1}$ belongs to $L^{\infty}(] 0, T\left[, L^{2}\left(\mathbb{R}^{-}\right)\right)$for $|\alpha| \leq 1$ and that $h_{1}$ and $h_{1}^{\prime}$ are in $L^{\infty}(] 0, T[)$ by taking $v^{2}=0$. Similarly (6.9) implies that $\partial^{\alpha} g_{2}$ is in $L^{\infty}(] 0, T\left[, L^{2}\left(\mathbb{R}^{-}\right)\right)$for $|\alpha| \leq 1$ and that $h_{1}$ and $h_{1}^{\prime}$ are in $L^{\infty}(] 0, T[)$ by taking $v^{1}=0$.

Thus, it remains to prove (6.9). Finally, we estimate $\left\|\partial^{\alpha}\left(g_{1}-g_{2}\right)\right\|_{L^{\infty}(] 0, T\left[, L^{2}\left(\mathbb{R}^{-}\right)\right)}$, $|\alpha| \leq 1,\left\|h_{1}-h_{2}\right\|_{L^{\infty}(] 0, T[)}$ and $\left\|h_{1}^{\prime}-h_{2}^{\prime}\right\|_{L^{\infty}(] 0, T[)}$ using the Mean Value Theorem for $f$ and its derivatives and for the Sobolev injection of $H^{1}\left(\mathbb{R}^{-}\right)$in $L^{\infty}\left(\mathbb{R}^{-}\right)$.

Corollary 2. Let $v$ be such that $\partial^{\alpha} v$ is in $L^{\infty}\left(10, T\left[, L^{2}\left(\mathbb{R}^{-}\right)\right)\right.$for $|\alpha| \leq 2, v(0,)=$. $u_{0}$ and $\partial_{t} v(0,)=.u_{1}$, where $u_{0} \in H^{2}\left(\mathbb{R}^{-}\right)$and $u_{1} \in H^{1}\left(\mathbb{R}^{-}\right)$have compact support in $\mathbb{R}^{-}$. Let $u$ be the unique solution of Proposition 5 with $g=f(J v)+v$ and $h=\partial_{t}^{-1} f_{1}$, where $f_{1}=f_{1}(J v)$. Then, $u$ satisfies the following estimate:

$$
\begin{array}{rl}
\max _{|\alpha| \leq 2}\left\|\partial^{\alpha} u\right\|_{L^{\infty}(] 0, T\left[, L^{2}\left(\mathbb{R}^{-}\right)\right)}^{2} & C e^{T}\left(T\left(1+T^{2}\right)(r \theta)^{2}\left(\max _{|\alpha| \leq 2}\left\|\partial^{\alpha} v\right\|_{L^{\infty}(] 0, T\left[, L^{2}\left(\mathbb{R}^{-}\right)\right)}\right)\right. \\
& \left.+\theta_{0}\left(\left\|u_{0}\right\|_{H^{2}\left(\mathbb{R}^{-}\right)}+\left\|u_{1}\right\|_{H^{1}\left(\mathbb{R}^{-}\right)}\right)\right),
\end{array}
$$


where $C$ is a universal constant and $\theta$ and $\theta_{0}$ are increasing functions depending on $f$.

Proof. Taking $v^{1}=v$ and $v^{2}=0$ in Lemma 1 yields

$$
\begin{aligned}
& \max _{|\alpha| \leq 1}\left\|\partial^{\alpha} g\right\|_{L^{\infty}(] 0, T\left[, L^{2}\left(\mathbb{R}^{-}\right)\right)}+\|h\|_{L^{\infty}(] 0, T[)}+\left\|h^{\prime}\right\|_{L^{\infty}(] 0, T[)} \\
& \leq \sqrt{1+T^{2}}(r \theta)\left(\max _{|\alpha| \leq 2}\left\|\partial^{\alpha} v\right\|_{L^{\infty}(] 0, T\left[L^{2}\left(\mathbb{R}^{-}\right)\right)}\right) .
\end{aligned}
$$

Moreover, $g(0,)=.f\left(0, . . u_{0}, u_{1}, \partial_{x} u_{0}\right)+u_{0}$. As $H^{1}\left(\mathbb{R}^{-}\right)$is embedded in $L^{\infty}\left(\mathbb{R}^{-}\right)$, the properties of $f$ and the mean value theorem give the existence of an increasing function $\theta_{0}$ such that

$$
\left\|u_{0}\right\|_{H^{2}\left(\mathbb{R}^{-}\right)}^{2}+\left\|u_{1}\right\|_{H^{1}\left(\mathbb{R}^{-}\right)}^{2}+\|g(0, .)\|_{L^{2}\left(\mathbb{R}^{-}\right)}^{2} \leq \theta_{0}\left(\left\|u_{0}\right\|_{H^{2}\left(\mathbb{R}^{-}\right)}+\left\|u_{1}\right\|_{H^{1}\left(\mathbb{R}^{-}\right)}\right) .
$$

(6.10), (6.11) and (6.2) imply the result.

Let $C_{1}=2 C e \theta_{0}\left(\left\|u_{0}\right\|_{H^{2}\left(\mathbb{R}^{-}\right)}+\left\|u_{1}\right\|_{H^{1}\left(\mathbb{R}^{-}\right)}\right)$. Let $T_{1}=\left(4 C C_{1} \theta\left(C_{1}\right)^{2} e\right)^{-1}$ and $T_{2}=\left(12 \operatorname{Ce} \theta\left(2 C_{1}\right)^{2}\right)^{-1}$. Let $T>0$ be such that $T \leq \min \left(T_{1}, T_{2}, 1\right)$. We define by induction the sequence $\left(u^{n}\right)$ such that $\partial^{\alpha} u^{n}$ is in $L^{\infty}(] 0, T\left[, L^{2}\left(\mathbb{R}^{-}\right)\right)$for $|\alpha| \leq 2$. We first take $u^{0}(t, x)=0$. Suppose that $u^{n}$ is such that $\partial^{\alpha} u^{n}$ is in $L^{\infty}(] 0, T\left[, L^{2}\left(\mathbb{R}^{-}\right)\right)$ for $|\alpha| \leq 2$. Let $g_{n}=f\left(., ., J u^{n}\right)+u^{n}$ and $h_{n}=\partial_{t}^{-1} f_{1}^{n}$, where $f_{1}^{n}=f_{1}\left(., J u^{n}\right)$. Then $g_{n}$ is in $H^{1}(] 0, T\left[\times \mathbb{R}^{-}\right), h_{n}$ belongs to $H^{1}(] 0, T[)$ by Lemma 1 and $h_{n}(0)=0$. Thus, using Proposition 5, we define $u^{n+1}$ as the unique solution of (6.1) with $g=g_{n}$ and $h=h_{n}$.

Lemma 2. $\left(\partial^{\alpha} u^{n}\right)$ is bounded and is a Cauchy sequence in $L^{\infty}(] 0, T\left[, L^{2}\left(\mathbb{R}^{-}\right)\right)$for $|\alpha| \leq 2$.

Proof. We prove by induction that there exists a constant $C_{1}$ such that for all $n \geq 0, u^{n}$ satisfies

$$
\max _{|\alpha| \leq 2}\left\|\partial^{\alpha} u^{n}\right\|_{L^{\infty}(] 0, T\left[, L^{2}(\Omega)\right)}^{2} \leq C_{1} .
$$

It is obviously true for $n=0$ since $u^{0}=0$. Suppose it is true for $n \geq 0$. Then, Corollary 2 implies

$$
\begin{aligned}
& \max _{|\alpha| \leq 2}\left\|\partial^{\alpha} u^{n+1}\right\|_{L^{\infty}(] 0, T\left[L^{2}\left(\mathbb{R}^{-}\right)\right)}^{2} \\
& \leq C e^{T}\left(T\left(1+T^{2}\right) C_{1}^{2} \theta^{2}\left(C_{1}\right)+\theta_{0}\left(\left\|u_{0}\right\|_{H^{2}\left(\mathbb{R}^{-}\right)}+\left\|u_{1}\right\|_{H^{1}\left(\mathbb{R}^{-}\right)}\right)\right) .
\end{aligned}
$$

As $T \leq 1$ and by definition of $C_{1}$,

$$
\max _{|\alpha| \leq 2}\left\|\partial^{\alpha} u^{n+1}\right\|_{L^{\infty}(] 0, T\left[, L^{2}\left(\mathbb{R}^{-}\right)\right)}^{2} \leq 2 C e T C_{1}^{2} \theta^{2}\left(C_{1}\right)+\frac{C_{1}}{2},
$$

which implies (6.12) for $n+1$ as $T \leq T_{1}$. Therefore, the sequence $\left(\partial^{\alpha} u^{n}\right)$ is bounded in $L^{\infty}(] 0, T\left[, L^{2}\left(\mathbb{R}^{-}\right)\right)$for $|\alpha| \leq 2$.

Let us show that $\left(\partial^{\alpha} u^{n}\right)$ is a Cauchy sequence in $L^{\infty}(] 0, T\left[, L^{2}\left(\mathbb{R}^{-}\right)\right)$for $|\alpha| \leq 2$. Let $n \geq 1, g_{n}=f\left(., ., J u^{n}\right)+u^{n}-f\left(., ., J u^{n-1}\right)-u^{n-1}$ and $h_{n}=\partial_{t}^{-1}\left(f_{1}^{n}-f_{1}^{n-1}\right)$. Then $u^{n+1}-u^{n}$ is the solution of (6.1) with $g=g_{n}, h=h_{n}$ and vanishing initial data. Moreover, $g_{n}(0,)=$.0 . (6.2) yields

$$
\max _{|\alpha| \leq 2}\left\|\partial^{\alpha}\left(u^{n+1}-u^{n}\right)\right\|_{L^{\infty}(] 0, T\left[, L^{2}\left(\mathbb{R}^{-}\right)\right)}^{2} \leq C e^{T}\left(\left\|g_{n}\right\|_{H^{1}(] 0, T\left[\times \mathbb{R}^{-}\right)}^{2}+\left\|h_{n}\right\|_{H^{1}(] 0, T[)}^{2}\right) .
$$


Estimates (6.9) and (6.12) imply

$$
\begin{aligned}
& \left\|g_{n}\right\|_{H^{1}(] 0, T\left[\times \mathbb{R}^{-}\right)}^{2}+\left\|h_{n}\right\|_{H^{1}(] 0, T[)}^{2} \\
& \leq 3 T\left(\max _{|\alpha| \leq 1}\left\|\partial^{\alpha} g_{n}\right\|_{L^{\infty}(] 0, T\left[, L^{2}\left(\mathbb{R}^{-}\right)\right)}^{2}+\left\|h_{n}\right\|_{L^{\infty}(] 0, T[)}^{2}+\left\|h_{n}^{\prime}\right\|_{\left.\left.L^{\infty}(] 0, T\right]\right)}^{2}\right) \\
& \leq 6 T \theta\left(2 C_{1}\right)^{2} \max _{|\alpha| \leq 2}\left\|\partial^{\alpha}\left(u^{n}-u^{n-1}\right)\right\|_{L^{\infty}(] 0, T\left[, L^{2}\left(\mathbb{R}^{-}\right)\right)}^{2},
\end{aligned}
$$

which together with (6.13) and $T \leq T_{2}$ yields

$$
\max _{|\alpha| \leq 2}\left\|\partial^{\alpha}\left(u^{n+1}-u^{n}\right)\right\|_{L^{\infty}(] 0, T\left[, L^{2}\left(\mathbb{R}^{-}\right)\right)}^{2} \leq \frac{1}{2} \max _{|\alpha| \leq 2}\left\|\partial^{\alpha}\left(u^{n}-u^{n-1}\right)\right\|_{L^{\infty}(] 0, T\left[, L^{2}\left(\mathbb{R}^{-}\right)\right)}^{2} .
$$

Therefore, there exists a constant $C_{3}$ such that

$$
\max _{|\alpha| \leq 2}\left\|\partial^{\alpha}\left(u^{n+1}-u^{n}\right)\right\|_{L^{\infty}(] 0, T\left[, L^{2}\left(\mathbb{R}^{-}\right)\right)}^{2} \leq \frac{C_{3}}{2^{n}} .
$$

Thus, $\left(\partial^{\alpha} u^{n}\right)$ is a Cauchy sequence in $L^{\infty}(] 0, T\left[, L^{2}\left(\mathbb{R}^{-}\right)\right)$for $|\alpha| \leq 2$.

Proof of Theorem 1. Let $u$ be the limit of the Cauchy sequence $\left(u^{n}\right)$. $\partial^{\alpha} u$ is in $L^{\infty}(] 0, T\left[, L^{2}\left(\mathbb{R}^{-}\right)\right)$for $|\alpha| \leq 2$. (6.9) and (6.12) yield

$$
\begin{aligned}
& \max _{|\alpha| \leq 1}\left\|\partial^{\alpha}\left(g_{n}-g\right)\right\|_{L^{\infty}(] 0, T\left[, L^{2}\left(\mathbb{R}^{-}\right)\right)}+\left\|h_{n}-h\right\|_{L^{\infty}(] 0, T[)}+\left\|h_{n}^{\prime}-h^{\prime}\right\|_{L^{\infty}(] 0, T[)} \\
& \leq \sqrt{2} \theta\left(2 C_{1}\right) \max _{|\alpha| \leq 2}\left\|\partial^{\alpha}\left(u^{n}-u\right)\right\|_{L^{\infty}(] 0, T\left[, L^{2}\left(\mathbb{R}^{-}\right)\right)},
\end{aligned}
$$

where $g_{n}=f\left(., ., J u^{n}\right), g=f(., ., J u), h_{n}=\partial_{t}^{-1} f_{1}^{n}$ and $h=\partial_{t}^{-1} f_{1}$ where $f_{1}=$ $f_{1}(., J u)$. (6.16) implies the convergence of the nonlinear terms, and we use the weak convergence in $L^{\infty}(] 0, T\left[, H^{1}\left(\mathbb{R}^{-}\right)\right)$for the linear terms.

Suppose there are two solutions $u^{1}$ and $u^{2}$ with the same initial boundary conditions satisfying the hypothesis of Theorem 10 Then, $u=u^{1}-u^{2}$ satisfies (6.1) with $u_{0}=0, u_{1}=0, g=f\left(., ., J u^{1}\right)+u^{1}-f\left(., ., J u^{2}\right)-u^{2}$ and $h=\partial_{t}^{-1}\left(f_{1}^{1}-f_{1}^{2}\right)$, where $f_{1}^{j}=f_{1}\left(., J u^{j}\right)$ for $j=1,2$. Moreover, $g(0,)=$.0 . Energy estimate (6.2) yields

$$
\max _{|\alpha| \leq 2}\left\|\partial^{\alpha} u\right\|_{L^{\infty}(] 0, T\left[, L^{2}\left(\mathbb{R}^{-}\right)\right)}^{2} \leq C e^{T}\left(\|g\|_{H^{1}(] 0, T\left[\times \mathbb{R}^{-}\right)}^{2}+\|h\|_{H^{1}(] 0, T[)}^{2}\right) .
$$

Using (6.9) as we did to get (6.14), we obtain a constant $C_{4}$ such that

$$
\max _{|\alpha| \leq 2}\left\|\partial^{\alpha} u\right\|_{L^{\infty}(] 0, T\left[, L^{2}\left(\mathbb{R}^{-}\right)\right)}^{2} \leq C_{4} T \max _{|\alpha| \leq 2}\left\|\partial^{\alpha} u\right\|_{L^{\infty}(] 0, T\left[, L^{2}\left(\mathbb{R}^{-}\right)\right)}^{2},
$$

which implies $u=0$ if $T<\frac{1}{C_{4}}$. Therefore, we get the local uniqueness which yields the global uniqueness.

\section{Numerical RESUlts}

7.1. The frame. We take the interval $] 0,2[$ as computational domain. We choose the stepsizes small in order to see the errors due to the various boundary conditions and not to the discretization: $\delta t=0.01$ and $h=0.001$. We choose $u_{0}$ in $H^{4}(\mathbb{R})$ and $u_{1}$ in $H^{3}(\mathbb{R})$ with compact support in $] 0,2[$ :

$$
\left\{\begin{array}{l}
\left.u_{0}(x)=x^{3}(2-x)^{3} \text { on }\right] 0,2[, \\
\left.\left.u_{0}(x)=0 \text { on }\right]-\infty, 0\right] \cup[2,+\infty[, \\
\left.u_{1}(x)=3 x^{2}(2-x)^{2}(x-1) \text { on }\right] 0,2[, \\
\left.\left.u_{1}(x)=0 \text { on }\right]-\infty, 0\right] \cup[2,+\infty[.
\end{array}\right.
$$

The solution of the nonlinear problem in $\mathbb{R}(2.1)$ propagates at speed 1 (see for instance 18]). In order to compute this solution with initial data (7.1) and for $t$ 
between 0 and 10, we compute the equation in ] - 10,12[ with Dirichlet boundary conditions.

Remark. In order to implement the semilinear wave equation equation with the absorbing boundary conditions, the support of the initial data must be included in the computational domain. In order to implement the method using the Dirichlet boundary conditions, the support of the solution must be included in the computational domain on the whole time interval. Therefore, the method using the absorbing boundary conditions approaches problem (2.1) with a low numerical cost compared to the method using Dirichlet boundary conditions.

We want to compute the solution of

$$
\left\{\begin{array}{l}
\left.\left(\partial_{t}^{2}-\partial_{x}^{2}\right) u=f\left(u, \partial_{t} u, \partial_{x} u\right) \text { in }\right] 0, T[\times] 0,2[, \\
\partial_{t}^{l} \partial_{\nu} u+\partial_{t}^{l+1} u+f_{1}(J u)=0, \text { at } x=0 \text { and } x=2, \\
u=u_{0}, \partial_{t} u=u_{1} \text { at } t=0,
\end{array}\right.
$$

where $l=0$ or $l=1, \partial_{\nu}=\partial_{x}$ at $x=2$ and $\partial_{\nu}=-\partial_{x}$ at $x=0$. We restrict ourselves to $f_{1}$, corresponding to one of the boundary conditions of section 2, We use a finite difference scheme with $N=1 / h, x_{j}=j h$ for $0 \leq j \leq N$, and $t_{n}=n \delta t$. Inside the computational domain, $u_{j}^{n}$ is an approximation of $u\left(t_{n}, x_{j}\right)$, and we approximate the equation by

$$
\begin{aligned}
\frac{u_{j}^{n+1}-2 u_{j}^{n}+u_{j}^{n-1}}{(\delta t)^{2}} & -\frac{u_{j+1}^{n+1}-2 u_{j}^{n+1}+u_{j-1}^{n+1}+u_{j+1}^{n-1}-2 u_{j}^{n-1}+u_{j-1}^{n-1}}{2 h^{2}} \\
& =f\left(u_{j}^{n}, v_{j}^{n}, \frac{u_{j+1}^{n}-u_{j-1}^{n}}{2 h}\right),
\end{aligned}
$$

where $1 \leq j \leq N-1$, and $v_{j}^{n}$ is defined by

$$
\begin{aligned}
& v_{j}^{1}=\frac{u_{j}^{1}-u_{j}^{0}}{\delta t}, \\
& v_{j}^{n}=\frac{3 u_{j}^{n}-4 u_{j}^{n-1}+u_{j}^{n-2}}{2 \delta t} \text { for } n \geq 2 .
\end{aligned}
$$

In cases (2.3), (2.4) and (2.6), the boundary condition has the form $\partial_{\nu} u+\partial_{t} u+$ $f_{1}\left(u, \partial_{t} u, \partial_{x} u\right)=0$ and is approximated by

$$
\frac{u_{0}^{n+1}+u_{0}^{n-1}-u_{2}^{n+1}-u_{2}^{n-1}}{4 h}+\frac{u_{1}^{n+1}-u_{1}^{n-1}}{2 \delta t}+f_{1}\left(u_{1}^{n}, v_{1}^{n}, \frac{u_{2}^{n}-u_{0}^{n}}{2 h}\right)=0
$$

and

$$
\begin{array}{r}
\frac{u_{N}^{n+1}+u_{N}^{n-1}-u_{N-2}^{n+1}-u_{N-2}^{n-1}}{4 h}+\frac{u_{N-1}^{n+1}-u_{N-1}^{n-1}}{2 \delta t} \\
\quad+f_{1}\left(u_{N-1}^{n}, v_{N-1}^{n}, \frac{u_{N}^{n}-u_{N-2}^{n}}{2 h}\right)=0 .
\end{array}
$$

In cases (2.5) and (2.7), the boundary condition has the form $\partial_{t} \partial_{\nu} u+\partial_{t}^{2} u+$ $f_{1}\left(u, \partial_{t} u, \partial_{x} u\right)=0$ and is approximated by

$$
\begin{gathered}
\frac{u_{0}^{n+1}-u_{0}^{n-1}-u_{1}^{n+1}+u_{1}^{n-1}}{2 h \delta t}+1 / 2\left(\frac{u_{0}^{n+1}-2 u_{0}^{n}+u_{0}^{n-1}}{(\delta t)^{2}}+\frac{u_{1}^{n+1}-2 u_{1}^{n}+u_{1}^{n-1}}{(\delta t)^{2}}\right) \\
+f_{1}\left(\frac{u_{1}^{n}+u_{0}^{n}}{2}, \frac{v_{1}^{n}+v_{0}^{n}}{2}, \frac{u_{1}^{n}-u_{0}^{n}}{h}\right)=0
\end{gathered}
$$


and by

$$
\begin{gathered}
\frac{u_{N}^{n+1}-u_{N}^{n-1}-u_{N-1}^{n+1}+u_{N-1}^{n-1}}{2 h \delta t}+\frac{1}{2}\left(\frac{u_{N}^{n+1}-2 u_{N}^{n}+u_{N}^{n-1}}{(\delta t)^{2}}+\frac{u_{N-1}^{n+1}-2 u_{N-1}^{n}+u_{N-1}^{n-1}}{(\delta t)^{2}}\right) \\
+f_{1}\left(\frac{u_{N-1}^{n}+u_{N}^{n}}{2}, \frac{v_{N-1}^{n}+v_{N}^{n}}{2}, \frac{u_{N}^{n}-u_{N-1}^{n}}{h}\right)=0 .
\end{gathered}
$$

Schemes (7.2) (7.4) (7.5), and (7.2) (7.6) (77.7) are semi-implicit (i.e. the implicit part is linear), and the error of consistency is second-order in space and time.

7.2. Comments on the results. We call relative error in the $L^{2}$ norm at time $t$ the expression

$$
\frac{\left\|u(t, .)-u_{e x}(t, .)\right\|_{L^{2}}}{\left\|u_{0}\right\|_{L^{2}}+\left\|u_{1}\right\|_{L^{2}}}
$$

where $u_{e x}$ is the solution of (2.1), $u$ is the solution computed with one of the various absorbing boundary conditions, and where we take the $L^{2}$ norm on the interval $] 0.02,1.98[$ which is included in the computational domain.

7.2.1. The linear case. We first give some results obtained in the linear case with the strategy of section 3 . We give the relative error in the $L^{2}$ norm for times between 0 and 10 in two cases. We compute the solution of equation $\partial_{t}^{2} u-\partial_{x}^{2} u=-\partial_{t} u$ (see Figure 1), and we show the results obtained for the condition of order 0 , the first-order condition and the second-order condition given by (3.4) and (3.7). We compute the solution of equation $\partial_{t}^{2} u-\partial_{x}^{2} u=-\partial_{x} u$ (see Figure 2), and we show the results obtained for the condition of order 0 , the first-order condition and the second-order condition. We note an improvement for small times by increasing the order, which is consistent with the fact that the boundary conditions are obtained using high frequency expansions. In the case of Figure1, the first-order condition is

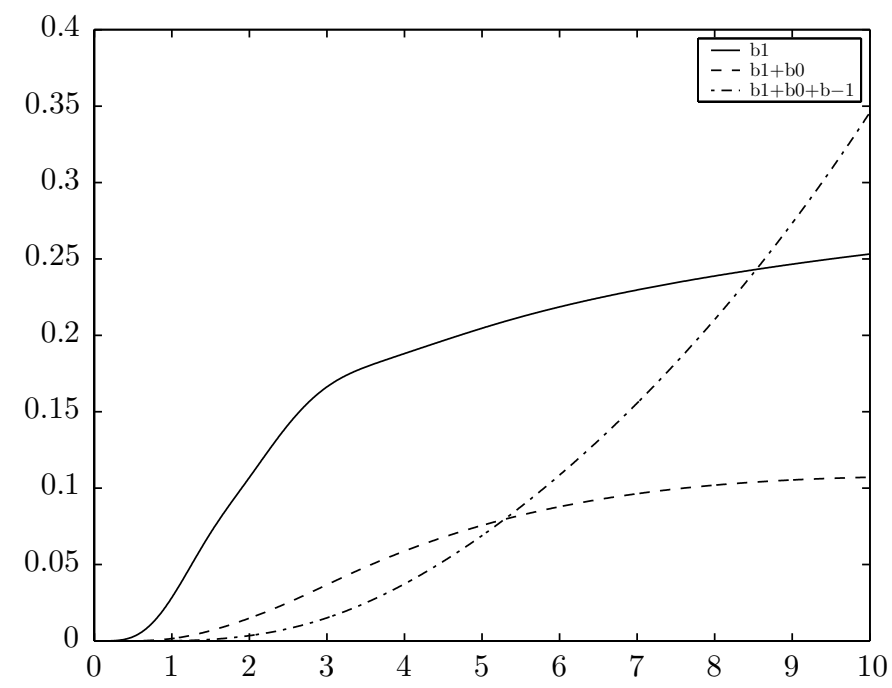

Figure 1. Relative error in the $L^{2}$ norm as a function of time. $\partial_{t}^{2} u-\partial_{x}^{2} u=-\partial_{t} u$. 


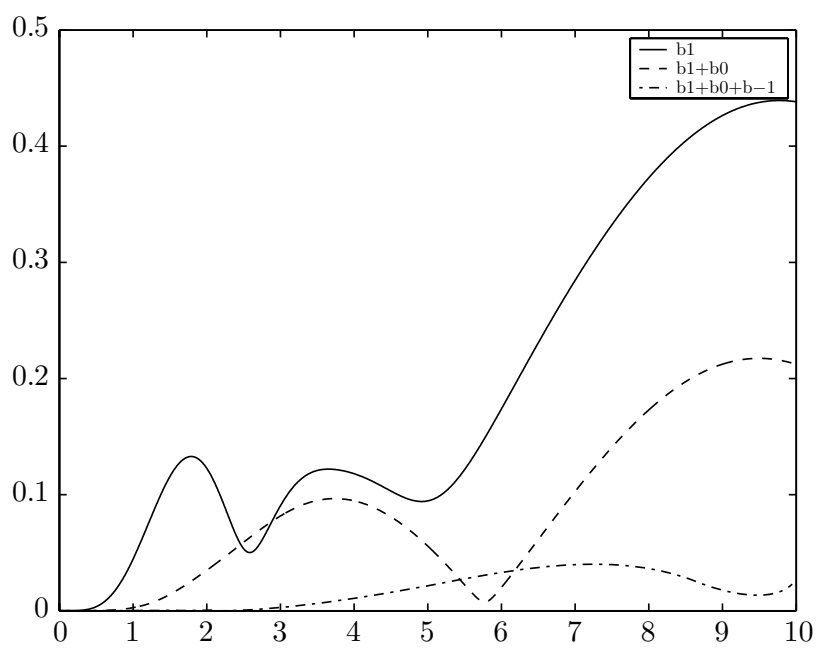

Figure 2. Relative error in the $L^{2}$ norm as a function of time. $\partial_{t}^{2} u-\partial_{x}^{2} u=-\partial_{x} u$.

more efficient than the condition of order 0 for all computed times, but the secondorder condition is more efficient than the first-order condition only for $t \leq 5.2$. In the case of Figure 2, the first-order condition is more efficient than the condition of order 0 except around $t=2.5$, and the second-order condition is more efficient than the first-order condition except around $t=5.75$.

7.2.2. The nonlinear case with $f\left(u, \partial_{t} u, \partial_{x} u\right)=-u^{2} \partial_{t} u$. In the nonlinear case, we obtain the same kind of behavior. In Figure 3, we give the relative error in the $L^{2}$ norm for times between 0 and 10 . We give the results obtained with the paradifferential strategy for the condition of order 0 , the first-order condition and the second-order condition. We note an improvement by increasing the order, and

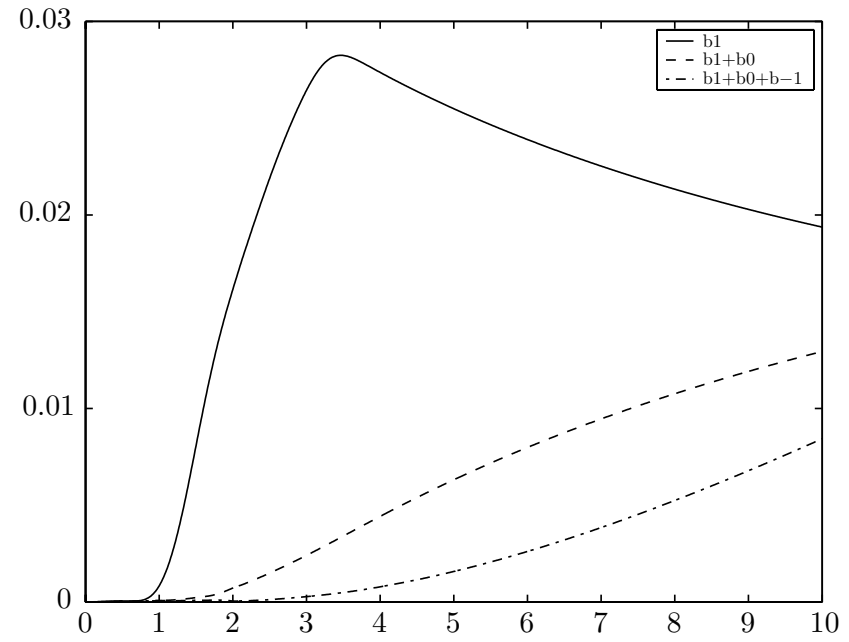

Figure 3. Relative error in the $L^{2}$ norm as a function of time. $f=-u^{2} \partial_{t} u$. 


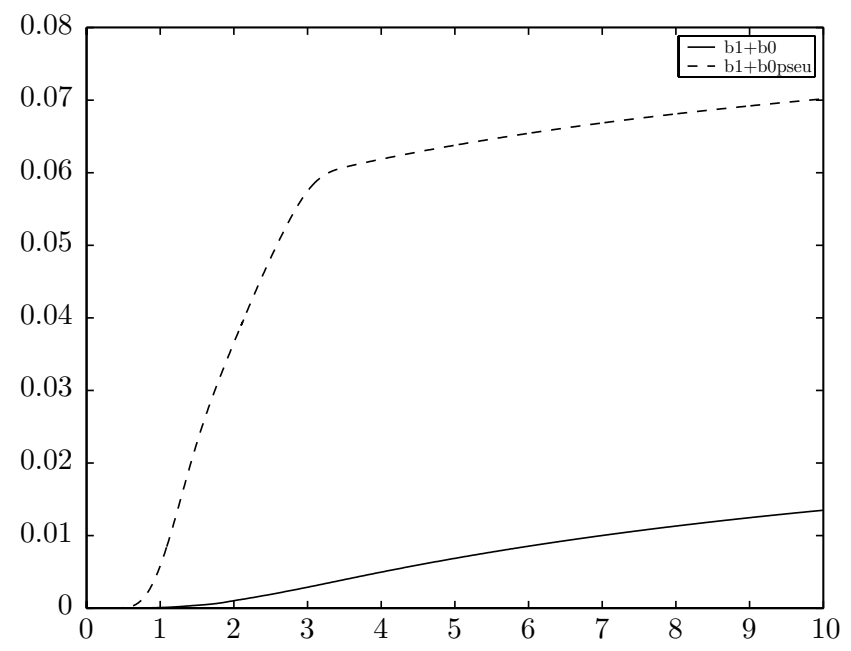

Figure 4. Relative error in the $L^{2}$ norm as a function of time. $f=-u^{2} \partial_{t} u$.

the results given by the second-order condition are very satisfactory: the error remains under $0.8 \%$ on the whole time interval $[0,10]$.

In Figure 4, we give the relative error in $L^{2}$ norm for times between 0 and 10 . We give the results obtained for the first-order conditions of Table 2. The first-order condition obtained with the paradifferential calculus is much more efficient than the first-order condition obtained with the pseudodifferential calculus.

In Figure 5, we give the relative error in the $L^{2}$ norm for times between 0 and 10 . We give the results obtained for the second-order conditions of Table2, The secondorder condition obtained with the paradifferential calculus is much more efficient than the second-order condition obtained with the pseudodifferential calculus.

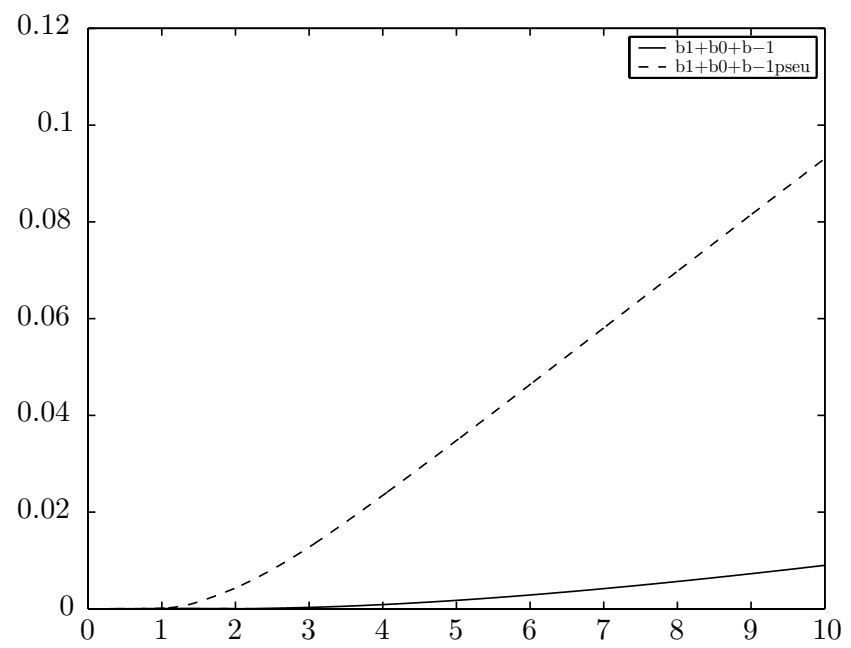

Figure 5. Relative error in the $L^{2}$ norm as a function of time. $f=-u^{2} \partial_{t} u$. 


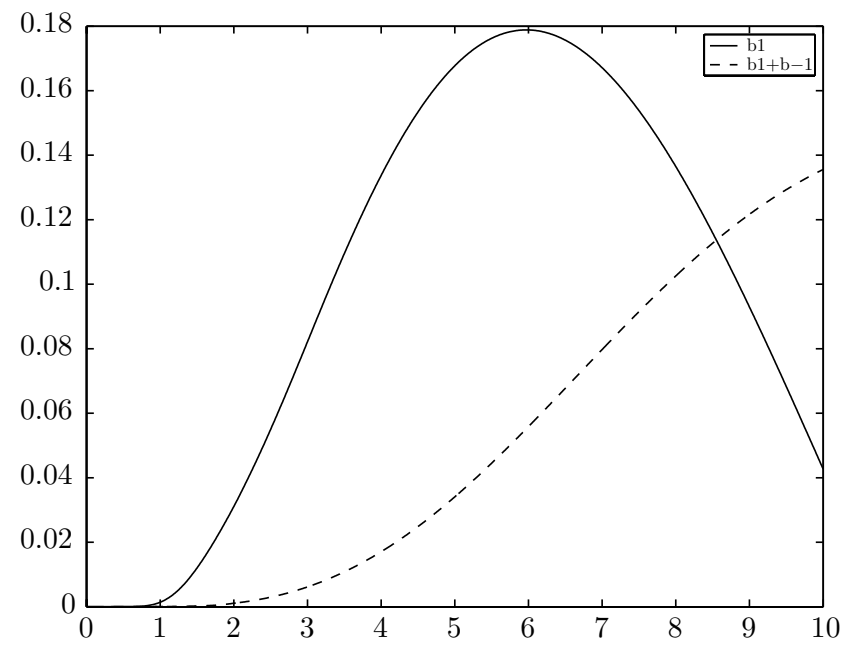

Figure 6. Relative error in the $L^{2}$ norm as a function of time. $f=-u^{3}$.

7.2.3. The nonlinear case with $f\left(u, \partial_{t} u, \partial_{x} u\right)=-u^{3}$. In Figure 6, we give the relative error in the $L^{2}$ norm for times between 0 and 10 . We give the results obtained for the zero-order condition and the second-order condition of Table 1 . We note an improvement by increasing the order for small times $(t \leq 8.5)$ as in the linear case.

7.2.4. The nonlinear case with $f\left(u, \partial_{t} u, \partial_{x} u\right)=u^{2} \partial_{x} u$. In Figure 7 we give the relative error in the $L^{2}$ norm for times between 0 and 10. We give the results obtained with the paradifferential strategy for the condition of order 0 , the first-order condition and the second-order condition. We note an improvement by increasing the order, and the results given by the second-order condition are very satisfactory: the error remains under $0.85 \%$ on the whole time interval $[0,10]$.

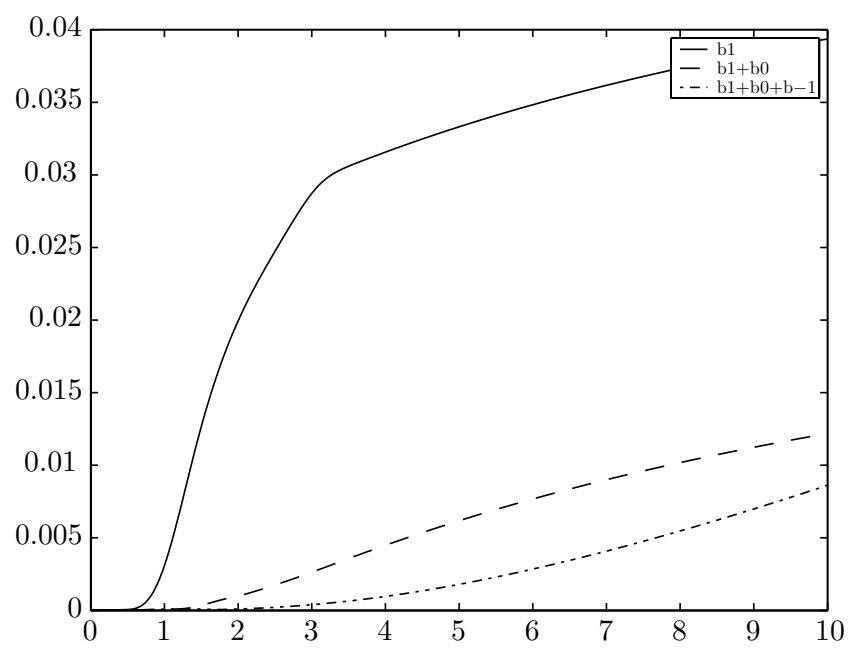

FIgURE 7. Relative error in the $L^{2}$ norm as a function of time. $f=u^{2} \partial_{x} u$. 


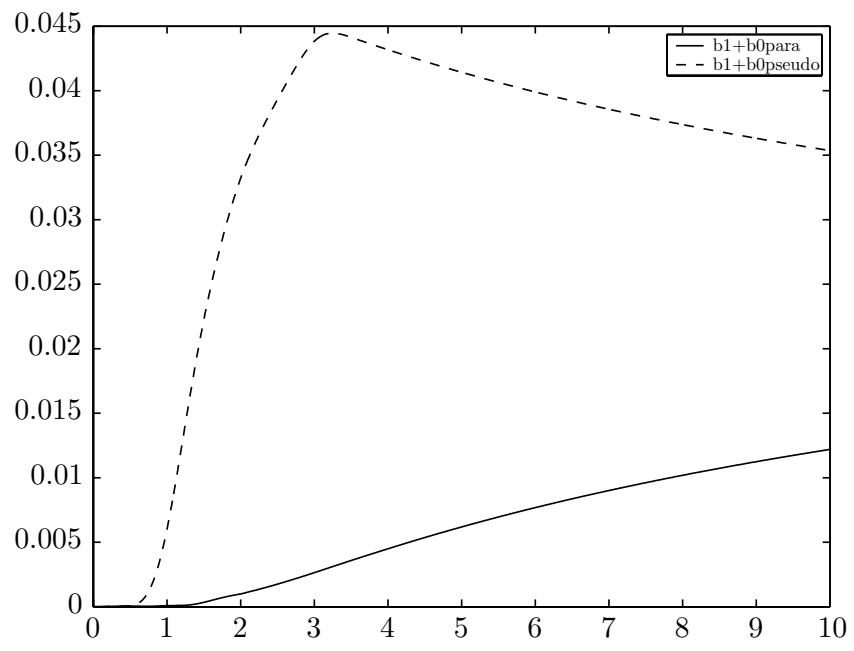

Figure 8. Relative error in the $L^{2}$ norm as a function of time. $f=u^{2} \partial_{x} u$.

In Figure 8, we give the relative error in $L^{2}$ norm for times between 0 and 10 . We give the results obtained for the first-order conditions of Table 3 . The first-order condition obtained with the paradifferential calculus is much more efficient than the first-order condition obtained with the pseudodifferential calculus.

In Figure 9, we give the relative error in the $L^{2}$ norm for times between 0 and 10. We give the results obtained for the second-order conditions of Table 3 , The second-order condition obtained with the paradifferential calculus is a little less efficient than the second-order condition obtained with the pseudodifferential calculus. However, both conditions are very satisfactory.

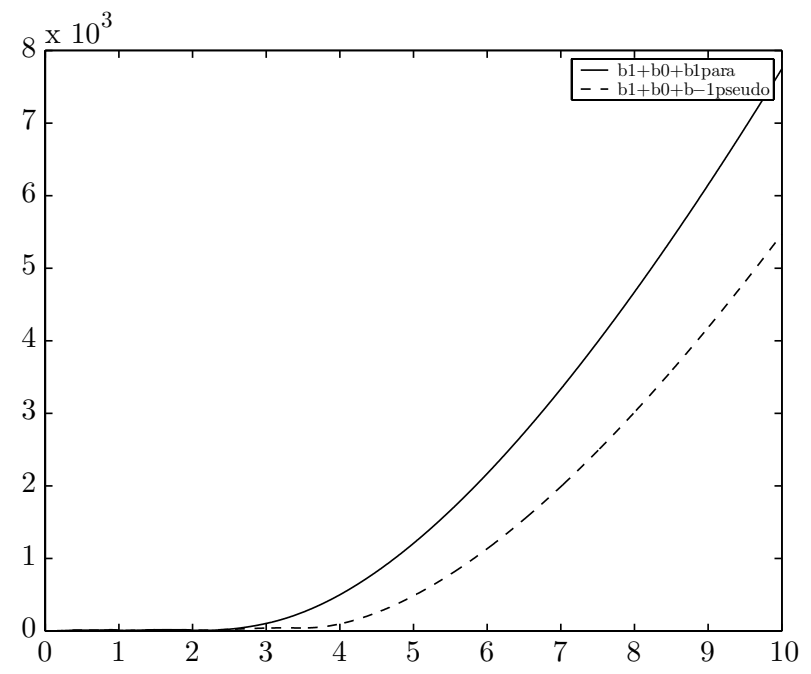

FiguRe 9. Relative error in the $L^{2}$ norm as a function of time. $f=u^{2} \partial_{x} u$. 


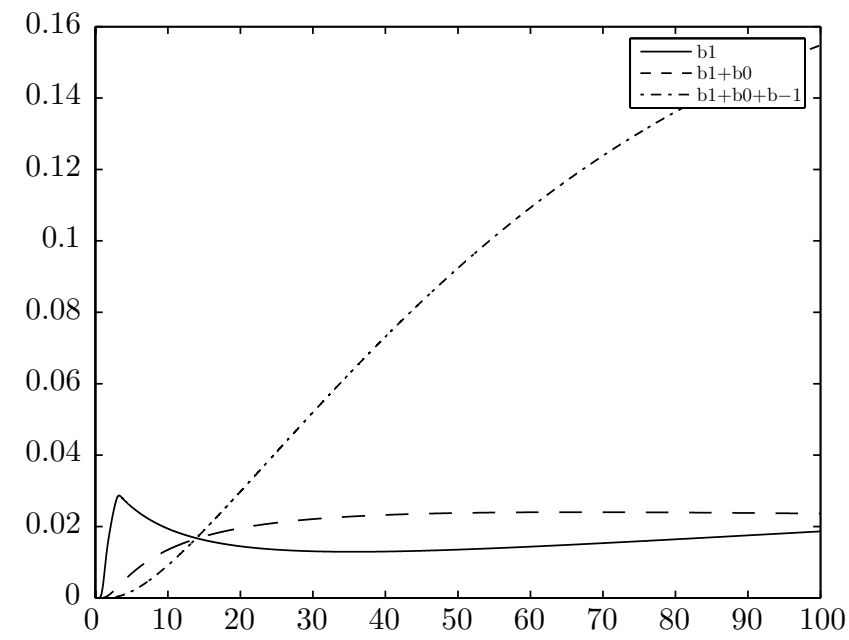

FIgURE 10. Long-time behavior. $f=-u^{2} \partial_{t} u$.

7.2.5. Long-time behavior. Now that we have proved the efficiency of the method on short-time intervals, we would like to investigate its long-time behavior. In Figure [10. we give the relative error in the $L^{2}$ norm for times between 0 and 100 . We choose $f\left(u, \partial_{t} u, \partial_{x} u\right)=-u^{2} \partial_{t} u$, and we give the results obtained with the paradifferential strategy for the condition of order 0 , the first-order condition and the second-order condition. While the second-order condition gives $15 \%$ error at time $t=100$, the zero and first-order conditions remain around $2 \%$ on the whole time interval. In Figure 11, we give the relative error in the $L^{2}$ norm for times between 0 and 100 .

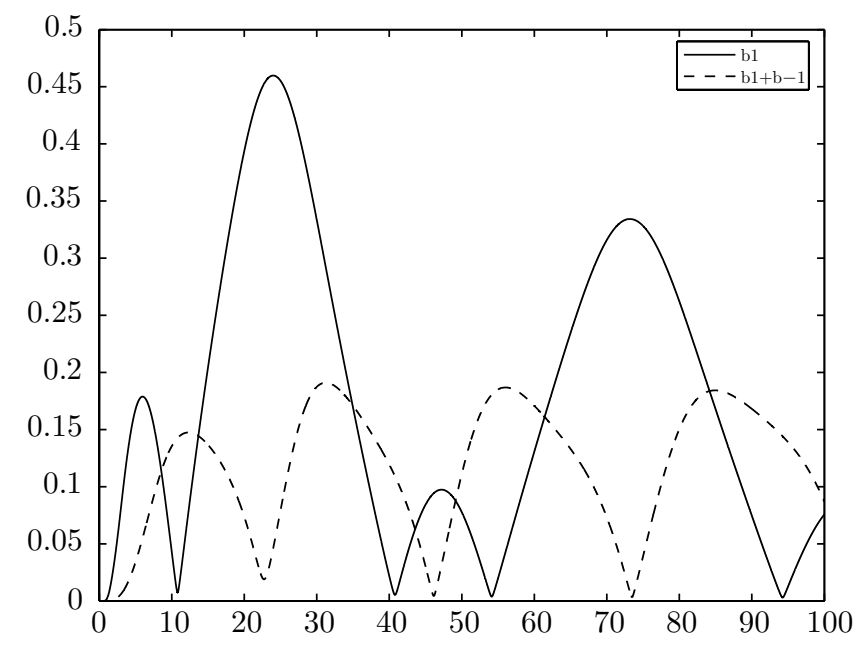

Figure 11. Long-time behavior. $f=-u^{3}$. 


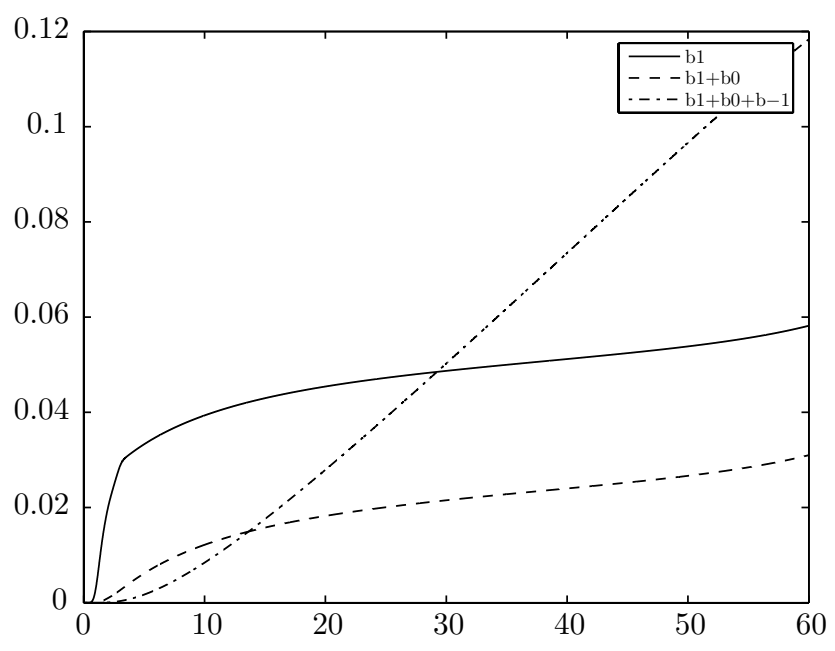

Figure 12. Long-time behavior. $f=u^{2} \partial_{x} u$.

We choose $f\left(u, \partial_{t} u, \partial_{x} u\right)=-u^{3}$, and we give the results obtained for the zero-order condition and the second-order condition of Table 1 . While the zero-order condition gives $45 \%$ error at time $t=24$, the second-order condition remains under $19 \%$ on the whole time interval. In Figure 12, we choose $f\left(u, \partial_{t} u, \partial_{x} u\right)=u^{2} \partial_{x} u$ and we give the results obtained with the paradifferential strategy for the condition of order 0 , the first-order condition and the second-order condition. We give the relative error in the $L^{2}$ norm for times between 0 and 60 , as our computations indicate that the solution of (2.1) blows up at $t=67$. While the second-order condition gives $12 \%$ error at time $t=100$, the zero- and first-order conditions remain respectively under $6 \%$ and $3 \%$ on the whole time interval. Although it seems hard to draw any general conclusion for the long-time behavior of our method, we note that in each case under study at least one of our absorbing boundary conditions performs well on long-time intervals.

Remark. When

$$
f\left(u, \partial_{t} u, \partial_{x} u\right)=-u^{2} \partial_{t} u
$$

or

$$
f\left(u, \partial_{t} u, \partial_{x} u\right)=-u^{3}
$$

the standard energy estimate for (2.1) implies a bound independant of time on $u(t,$.$) in H^{1}(\mathbb{R})$ and $\partial_{t} u(t,$.$) in L^{2}(\mathbb{R})$, which in turn yields global existence. When

$$
f\left(u, \partial_{t} u, \partial_{x} u\right)=u^{2} \partial_{x} u,
$$

the standard energy estimate for (2.1) does not imply such a bound due to the nonlinear contribution which has no sign, and it is therefore not surprising that a blow-up takes place in this case. 
TABLE 4. Maximum of the relative error in $L^{2}$ norm for times between 0 and 10 .

\begin{tabular}{|c|c|c|c|c|c|c|}
\hline$\varepsilon$ & 0.125 & 0.25 & 0.5 & 1 & 2 & 4 \\
\hline abc of order 0 & $0.74 \%$ & $2.9 \%$ & $10.3 \%$ & $15.4 \%$ & $49 \%$ & $47 \%$ \\
\hline second order abc of Table 1 1 & $0.02 \%$ & $0.06 \%$ & $0.67 \%$ & $6.6 \%$ & $5 \%$ & $37 \%$ \\
\hline
\end{tabular}

7.2.6. Dependence on the size of the initial data. Since the problem and the boundary conditions are nonlinear, we examine the dependence of the relative errors on the amplitude of the initial data. We consider a solution $u$ such that $u(0, x)=\varepsilon u_{0}(x)$ and $\partial_{t} u(0, x)=0$, where $\varepsilon>0$ and $u_{0}$ is given by section 7.1. In Table 4, we give the maximum of the relative error in the $L^{2}$ norm for times between 0 and 10 in function of $\varepsilon$. We choose $f\left(u, \partial_{t} u, \partial_{x} u\right)=-u^{3}$, and we give the results obtained for the zero-order condition and the second-order condition of Table 1. We notice an improvement by decreasing $\varepsilon$, and the second-order condition is much more efficient than the condition of order 0 .

7.2.7. Dependence on the size of the computational domain. Finally, we consider the influence of the size of the computational domain. For $\delta \geq 0$, we take $[-\delta, 2+\delta]$ as computational domain, and we define the distance as $100 . \delta / 2=50 . \delta$. We give the relative error in the $L^{2}$ norm for times between 0 and 10. In Figures 13 , 14 15. and 16, we take, respectively, a distance of $5 \%, 25 \%, 50 \%$, and $100 \%$. The results we obtained are similar to the three examples of nonlinearity. We choose $f\left(u, \partial_{t} u, \partial_{x} u\right)=-u^{2} \partial_{t} u$, and we give the results we obtained with the paradifferential strategy for the condition of order 0 , the first-order condition and the second-order condition. We note that increasing the distance gives the same result as taking a distance equal to zero and decreasing the computed time interval. This emphasizes the relevance of our strategy which is valid at least for small times.

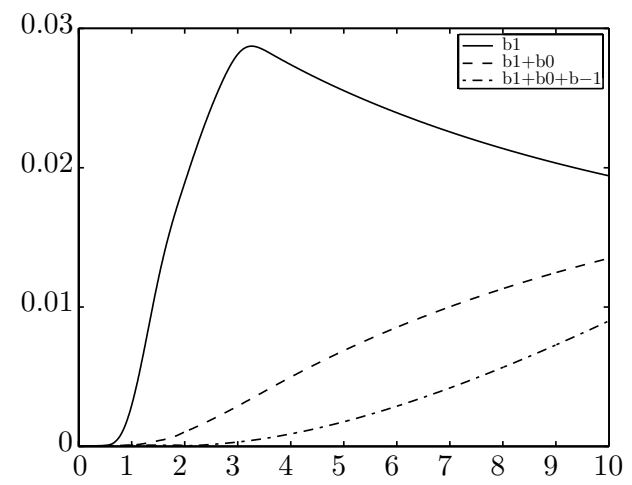

Figure 13. Distance to the boundary of $5 \%$.

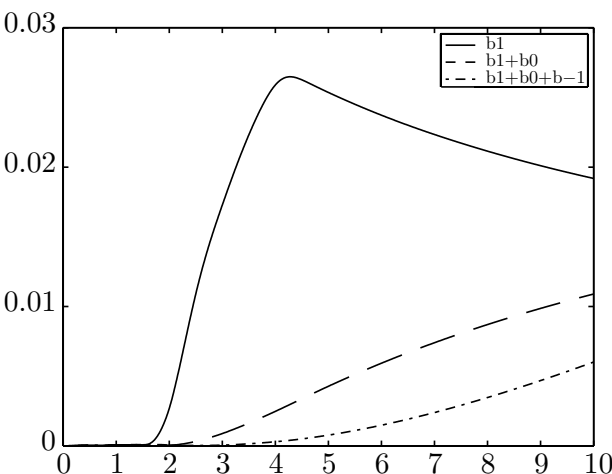

Figure 14. Distance to the boundary of $25 \%$. 


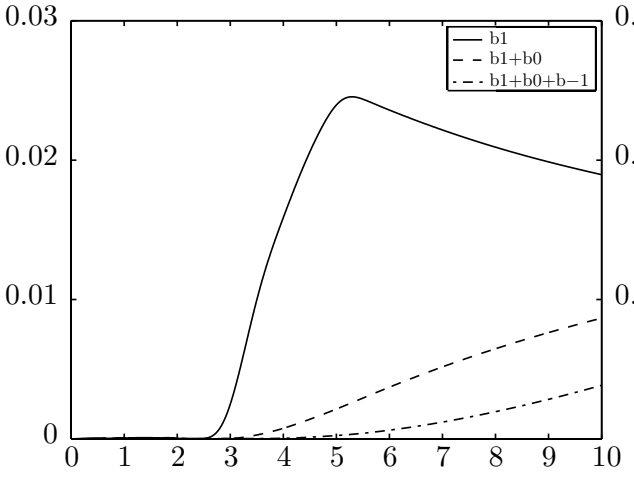

Figure 15. Distance to the boundary of $50 \%$.

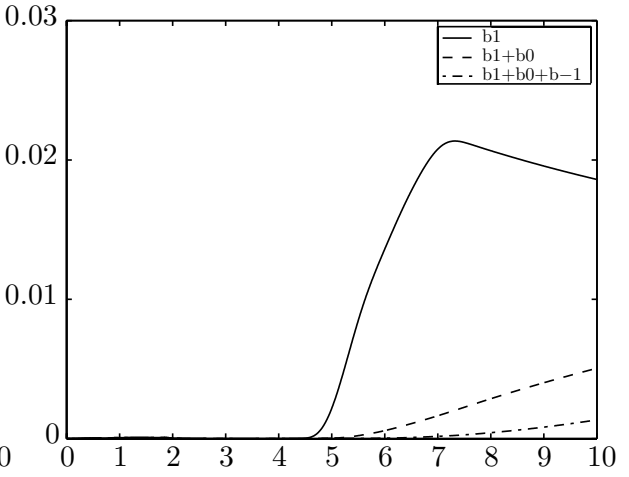

Figure 16. Distance to the boundary of $100 \%$.

\section{CONCLUSION}

We have constructed a hierarchy of absorbing boundary conditions for the semilinear wave equation. As in the linear case, we obtain an improvement of the precision of these boundary conditions by increasing the order except possibly for large times. Our strategy uses the paradifferential calculus and gives better results than the strategy using the pseudodifferential calculus. Thus, this method is efficient to approximate the semilinear wave equation on $\mathbb{R}$ with a low numerical cost.

\section{ACKNOWLEDGMENTS}

The author thanks the anonymous referees for many constructive comments which led to a great improvement of the paper.

\section{REFERENCES}

1. A. Alabidi, Réflexion transverse des singularités pour un problème aux limites non linéaire d'ordre 2, C. R. A. S, série I-10 t.300 (1985). MR0786901 (86m:35032)

2. B. Alpert, L. Greengard, T. Hagstrom, Rapid evaluation of nonreflecting boundary kernels for time-domain wave propagation, SIAM J. Numer. Anal. 37 (2000), 1138-1164. MR1756419 (2002c:65037)

3. J. P. Berenger, A perfectly matched layer for the absorption of electromagnetic waves, J. Comput. Phys. 114 (1994), 185-200. MR1294924 (95e:78002)

4. J. M. Bony, Calcul symbolique et propagation des singularités pour les équations aux dérivées partielles non linéaires, Ann. Sci. Ec. Norm. Sup (4 ème série). 14 (1981), 209-246. MR0631751 (84h:35177)

5. J. Y. Chemin, Fluides parfaits incompressibles, Astérisque 230 (1995). MR 1340046 (97d:76007)

6. E. Dubach, Nonlinear artificial boundary conditions for the viscous Burgers equation, prépublication 00/04 de l'université de Pau et des pays de l'Adour, 2000.

7. B. Engquist, A. Majda, Radiation boundary conditions for acoustic and elastic wave calculations, Comm. Pure and Appl. Math. 32 (1979), 313-357. MR0517938(80e:76041)

8. M. Grote, J. Keller, Exact nonreflecting boundary conditions for the time dependent wave equation, SIAM J. Appl. Math. 55 (1995), 280-297. MR.1322761 (95m:35125)

9. T. Hagstrom, Radiation boundary conditions for the numerical simulation of waves, Acta Numer. 8 (1999), 47-106. MR1819643(2002c:35171) 
10. L. Halpern, J. Rauch, Absorbing boundary conditions for diffusion equations, Numer. Math. 71 (1995), 185-224. MR1347164 (96h:65152)

11. L. Hörmander, Linear partial differential operators, Springer, Berlin, Heidelberg, 1969. MR $0248435(40: 1687)$

12. L. Hörmander, Lectures on nonlinear hyperbolic differential equations, Springer, Berlin, Heidelberg, 1997. MR1466700 (98e:35103)

13. E. L. Lindmann, Free-space boundary conditions for the time dependent wave equation, J. Comp. Phys. 18 (1975), 16-78.

14. A. Majda, S. Osher, Reflection of singularities at the boundary, Comm. Pure and Appl. Math. 28 (1975), 479-499. MR0492792 (58:11858a)

15. Y. Meyer, Remarques sur un théorème de J. M. Bony, Suppl. ai Rend. del Circolo mat. di Palermo (1981), 1-20. MR0639462 (83b:35169)

16. L. Nirenberg, Lectures on Linear Partial Differential Equations, CBMS Reg. Conf. 17, AMS, Providence, RI, 1976. MR0450755 (56:9048)

17. M. Sablé-Tougeron, Régularité microlocale pour des problèmes aux limites non linéaires, Ann. Inst. Fourier. 36 (1986), 39-82. MR.0840713 (88b:35021)

18. C. D. Sogge, Lectures on nonlinear wave equation, Monographs in Analysis, International Press, Boston, 1995. MR1715192 (2000g:35153)

19. J. Szeftel, Absorbing boundary conditions for reaction diffusion equation, IMA J. Appl. Math. 68 (2003), 167-184. MR1968310 (2004k:35219)

20. J. Szeftel, Design of absorbing boundary conditions for Schrödinger equations in $\mathbb{R}^{d}$, SIAM J. Numer. Anal. 42 (2004), 1527-1551. MR2114289

21. L. Trefethen, L. Halpern, Well-posedness of one-way wave equations and absorbing boundary conditions, Math. Comput. 47 (1986), 421-435. MR0856695 (88b:65148)

22. G. B. Whitham, Linear and nonlinear waves, John Wiley, New York, 1974. MR0483954 (58:3905)

LaGa UMr 7539, Institut Galilée, Université Paris 13, 99 Avenue J.B.Clément, 93430 Villetaneuse, France

E-mail address: szeftel@math.univ-paris.13.fr 